\title{
1 Morphogenesis and differentiation of embryonic vascular smooth muscle cells in \\ 2 zebrafish
}

3

4

Thomas R. Whitesell ${ }^{1,2}$, Paul Chrystal ${ }^{3,4}$, Jae-Ryeon Ryu ${ }^{1,2}$, Nicole Munsie ${ }^{1,2}$, Ann Grosse ${ }^{5}$, Curtis French ${ }^{4,6}$, Matthew L. Workentine ${ }^{7}$, Rui Li ${ }^{5}$, Lihua Julie Zhu ${ }^{5,8}$, Andrew Waskiewicz ${ }^{4}$, Ordan J. Lehmann³ ${ }^{3}$ Nathan D. Lawson ${ }^{5}$, Sarah J. Childs ${ }^{1,2 *}$

*corresponding author: Sarah Childs; schilds@ucalgary.ca; 403-220-8277

${ }^{1}$ Alberta Children's Hospital Research Institute, ${ }^{2}$ Department of Biochemistry and Molecular Biology, University of Calgary, 3330 Hospital Drive NW, Calgary, Alberta, Canada, T2N 4N1. ${ }^{3}$ Departments of Ophthalmology, and Medical Genetics, University of Alberta, Edmonton, Alberta, Canada. ${ }^{4}$ Department of Biological Sciences, CW405, Biological Sciences Bldg., 11455 Saskatchewan Dr., University of Alberta, Edmonton AB T6G 2E9; Women \& Children's Health Research Institute, ECHA 4-081, 1140587 Ave NW, University of Alberta, Edmonton, AB T6G 1C9; Neurosciences and Mental Health Institute, 4-120 Katz Group Centre, University of Alberta, Edmonton AB, T6G 2E1. 5 Department of Molecular, Cell, and Cancer Biology, University of Massachusetts Medical School, 364 Plantation Street, Worcester, MA, USA, 01605 ${ }^{6}$ Current address: Discipline of Genetics, Memorial University, St. John's, Newfoundland and Labrador, Canada. ${ }^{7}$ Faculty of Veterinary Medicine, University of Calgary, 3330 Hospital Drive NW, Calgary, Alberta, Canada, T2N 4N1. ${ }^{8}$ Program in Bioinformatics and Integrative Biology, 1 Program in Molecular Medicine, University of Massachusetts Medical School, Worcester, MA, USA, 01605 
Summary Statement: Tracing the morphogenesis and transcriptome of early vascular smooth muscle cells using foxc1b

Running title: Early smooth muscle cell development and transcriptome

Keywords: foxc1b, acta2, vascular smooth muscle cells, zebrafish, development, RNA-Seq transcriptome

\section{Abstract:}

42 Despite the critical role of vascular mural cells (smooth muscle cells and pericytes) in supporting 43 the endothelium of blood vessels, we know little of their early morphogenesis and differentiation. 44 foxc1b:EGFP expressing cells in zebrafish associate with the vascular endothelium ( $k d r l)$ and 45 co-express a smooth muscle marker (acta2), but not a pericyte marker (pdgfr $\beta$ ). The expression 46 of foxc1b in early peri-endothelial mesenchymal cells allows us to follow the morphogenesis of 47 mesenchyme into acta2 expressing vascular smooth muscle cells. We show that mural cells expressing different markers associate with vessels of different diameters, depending on their embryonic location and developmental timing, suggesting marker expression is predictive of functional differences. We identify gene expression signatures for an enriched vascular smooth

51 muscle cell population (foxc1b + acta2) and all smooth muscle (acta2) using fluorescence-

52 activated cell sorting and RNA-Seq. Finally, we demonstrate that progressive loss of

53 foxc1a/foxc1b results in decreased smooth muscle cell coverage. Together, our data highlight

54 the early cellular dynamics and transcriptome profiles of smooth muscle cells in vivo, using

55 foxc $1 b$ as a unique tool to probe vascular smooth muscle cell differentiation. 


\section{Introduction:}

Smooth muscle cells and pericytes (collectively known as vascular mural cells) encircle and stabilize the underlying endothelium, deposit extracellular matrix proteins, and provide contractility to blood vessels; however, they have distinct morphologies (Lindahl et al., 1997; Santoro, Pesce and Stainier, 2009; Stratman et al., 2009, 2017; Armulik, Genové and Betsholtz, 2011; Carmeliet and Jain, 2011; Whitesell et al., 2014). Vascular smooth muscle cells typically form a multilayer, continuous sheath on large caliber blood vessels subject to higher blood pressure. Found outside the basement membrane in mammals, vascular smooth muscle cells provide support and contractility to the underlying endothelium (Etchevers et al., 2001; Santoro, Pesce and Stainier, 2009; Olson and Soriano, 2011; Whitesell et al., 2014; Stratman et al., 2017). Pericytes are typically punctate, non-continuous cells with individual processes that wrap around smaller diameter blood vessels creating peg and socket connections with the underlying endothelium. Pericytes are essential in regulating vascular permeability, for example, in the bloodbrain barrier, and are more prevalent at branching points of blood vessels, turbulent areas of blood flow, or bends in complex blood vessels (Hellström et al., 1999; Etchevers et al., 2001; Bergers and Song, 2005; Winkler, Bell and Zlokovic, 2010; Armulik, Genové and Betsholtz, 2011; Olson and Soriano, 2011; Whitesell et al., 2014; Van Dijk et al., 2015; Craggs et al., 2015; Trost et al., 2016; He et al., 2016; Stratman et al., 2017; Birbrair et al., 2017; Dias Moura Prazeres et al., 2017; Berthiaume et al., 2018). Highlighting their importance, defects in vascular mural cells lead to cerebrovascular dysfunction including cerebral small vessel disease and stroke (Joutel et al., 1996; Craggs et al., 2014; French et al., 2014; Baron-Menguy et al., 2017). For example, loss of vascular smooth muscle cells results in thoracic aneurysm and dissections (TAAD) (Guo et al., 2009) and increased vessel size due to poor smooth muscle contraction (Abrams et al., 2016), while loss of pericytes leads to increased brain permeability, aneurysm, and hemorrhage (Lindahl et al., 1997; Moura, Lemos and Oliveira, 2017). Recent morphological and transcriptomic characterization in mouse suggests there are sub-types of pericytes and smooth muscle cells that vary in gene expression and morphology depending on their position in the vascular tree (Hartmann et al., 2015; Grant et al., 2017; Vanlandewijck et al., 2018). Further illustrating their complexity, mural cell subtypes are typically distinguished by morphology, location, contractile function, and molecular markers (Hill et al., 2015; He et al., 2016; Birbrair et al., 2017; Dias Moura Prazeres et al., 2017). In addition, there are multiple developmental origins of mural cells, as vascular smooth muscle cells and pericytes of the head arise from neural crest and mesoderm, while trunk vascular smooth muscle cells have a mesodermal origin (Etchevers et al., 2001; Pouget, Pottin and Jaffredo, 2008; Wang et al., 2014; Cavanaugh, Huang and Chen, 2015). 
Smooth muscle actin (acta2/asma) and transgelin (tagln/sm22 $\alpha-b)$ are the most widely used vascular smooth muscle markers (Solway et al., 1995; Hellström et al., 1999; Schildmeyer et al., 2000; Georgijevic et al., 2007; Santoro, Pesce and Stainier, 2009; Seiler, Abrams and Pack, 2010; Whitesell et al., 2014; Stratman et al., 2017). Pericytes on the other hand, are typically labelled with platelet-derived growth factor receptor-beta (pdgfr $\beta$ ) (Armulik, Genové and Betsholtz, 2011; Ando et al., 2016; He et al., 2016; Trost et al., 2016; Grant et al., 2017; Stratman et al., 2017; Berthiaume et al., 2018) or ng2/cspg4 (Armulik, Genové and Betsholtz, 2011; He et al., 2016; Trost et al., 2016; Underly et al., 2017; Berthiaume et al., 2018), in addition to tbx18 (Birbrair et al., 2017; Guimar??es-Camboa et al., 2017), notch3 (Wang et al., 2014; Henshall et al., 2015; Tao et al., 2017), nestin (Dias Moura Prazeres et al., 2017), rgs5 (Armulik, Genové and Betsholtz, 2011; He et al., 2016), abcc9 (He et al., 2016; Vanlandewijck et al., 2018), and desmin (Georgijevic et al., 2007; Armulik, Genové and Betsholtz, 2011; Trost et al., 2016). A key caveat is that even classic markers for smooth muscle cells (acta2, tagln) and pericytes (pdgfr $\beta$, ng2/cspg4), do not label every vascular mural cell (Santoro, Pesce and Stainier, 2009; Whitesell et al., 2014; Hartmann et al., 2015; He et al., 2016; Birbrair et al., 2017; Dias Moura Prazeres et al., 2017; Grant et al., 2017). Mural cell heterogeneity varies with location within an organism, cellular lineage, function, phenotype, and morphology (Georgijevic et al., 2007; Santoro, Pesce and Stainier, 2009; Armulik, Genové and Betsholtz, 2011; He et al., 2016; Dias Moura Prazeres et al., 2017), and consequently, heterogeneity in marker expression makes it difficult to identify a single marker for consistent labelling of vascular mural cells. In addition, there are a lack of smooth muscle markers during early development, particularly in zebrafish (Georgijevic et al., 2007), and the common pericyte markers have been shown to be expressed in numerous other cell types (He et al., 2016; Ivanova and Orekhov, 2016).

Cerebral pericytes are labelled by pdgfr $\beta$ expression as early as $48 \mathrm{hpf}$ (hours post fertilization) in zebrafish embryos (Wang et al., 2014), while ng2/cspg 4 is expressed by 72 hpf (Wang et al., 2014). In zebrafish, acta2 and tagln transgenic expression is first detectable around 3 days post fertilization (dpf), with robust vascular smooth muscle expression by $4 \mathrm{dpf}$ (Georgijevic et al., 2007; Majesky, 2007; Santoro, Pesce and Stainier, 2009; Seiler, Abrams and Pack, 2010; Whitesell et al., 2014; Gays et al., 2017). Using transmission electron microscopy, we previously observed mural cells with a mesenchymal phenotype associated with the endothelium as early as $48 \mathrm{hpf}$, and showed close association with endothelium correlating with vascular stabilization (Liu et al., 2007; Lamont et al., 2010). However, the earliest stages of vascular mural cell development in zebrafish cannot be observed using current transgenic markers. 
Since many vascular smooth muscle cells of the head are neural crest-derived, we focused on markers that label vascular mural cells early in development and are expressed in neural crest, such as the forkhead box domain transcription factor foxc1b. Loss of Foxc1 in mouse leads to severe defects in head mesenchyme, branchial arch and anterior somite development (Kume et al., 1998, 2001). In humans, it is also associated with anterior segment dysgenesis in the eye, and more recently, also with cerebral small vessel disease (Mears et al., 1998; Nishimura et al., 1998; French et al., 2014; Seo et al., 2017; Avasarala, Jones and Rogers, 2018). Mouse models show that Foxc1 mutants have disrupted mural cell contacts on the endothelium, and cerebral hemorrhage (Kume et al., 1998; Siegenthaler et al., 2013; French et al., 2014; Prasitsak et al., 2015; Mishra et al., 2016). Foxc1/c2 play important roles in numerous processes including lymphatic development (Seo et al., 2006; Van Steensel et al., 2009; Sasman et al., 2012; Fatima et al., 2016); vascular development (Kume et al., 2001; Yamagishi et al., 2003; Seo et al., 2006, 2012; De Val et al., 2008; Hayashi and Kume, 2008a, 2008b; Skarie and Link, 2009; Veldman and Lin, 2012; Koo and Kume, 2013; Prasitsak et al., 2015; Mishra et al., 2016); cardiac outflow tract development (Seo and Kume, 2006; Kodo et al., 2017); somitogenesis (Kume et al., 2001; J. M. Topczewska et al., 2001; Tamimi et al., 2006; Qiu et al., 2016); neural crest and skeletal development (Seo and Kume, 2006; Inman et al., 2013; Koo and Kume, 2013; Kodo et al., 2017; Seo et al., 2017); and hematopoiesis (Omatsu et al., 2014; Jang et al., 2015), and diseases such as glaucoma and Axenfeld-Rieger syndrome (Kume et al., 2001; Tamimi et al., 2006; Berry et al., 2008; Skarie and Link, 2009; French et al., 2014; Mishra et al., 2016; Souzeau et al., 2017). In mouse, Foxc1/c2 are expressed in neural crest (Kume et al., 1998; Seo et al., 2012, 2017; Siegenthaler et al., 2013), and in the endothelium (Kume et al., 1998, 2001; Koo and Kume, 2013). Foxc1 is also expressed in brain pericytes and smooth muscle cells and is co-expressed with Pdgfr $\beta$ and Acta2 as early as E14.5 (Zarbalis et al., 2007; Siegenthaler et al., 2013; Prasitsak et al., 2015; Mishra et al., 2016). In zebrafish, there are two foxc1 paralogs, foxc1a and foxc1b (J. M. Topczewska et al., 2001; Skarie and Link, 2009; Chen et al., 2017). foxc1b is first expressed during the involution of mesendoderm in early gastrulation, and by $33 \mathrm{hpf}$, foxc1b is expressed in the pharyngeal arch mesenchyme (Jolanta M. Topczewska et al., 2001). Knockdown of foxc1a/b in zebrafish shows a reduction in acta2 positive smooth muscle cells in the ventral aorta and aortic arch arteries (French et al., 2014), suggesting a role in mural cell development. Foxc1 expression is also reported in the endothelium of mouse and in the trunk endothelium of zebrafish (Kume et al., 1998; J. M. Topczewska et al., 2001; Skarie and Link, 2009; Siegenthaler et al., 2013; Prasitsak et al., 2015; Mishra et al., 2016; Winkler et al., 2018); however, the role of foxc1b and its targets in the vasculature are not yet well understood. 
Here, using a foxc1b:EGFP zebrafish transgenic reporter, we show that foxc $1 b$ labels smooth muscle cells and their precursors. We identify and visualize a key window for attachment of foxc1b:EGFP positive smooth muscle cells to the endothelium, and find smooth muscle differentiation is impaired in compound foxc1a/foxc1b mutants. The transcriptome of dual foxc $1 b$ + acta2 expressing smooth muscle cells uncovers a set of enriched genes specific to the embryonic vascular smooth muscle cell population during early differentiation.

\section{Results:}

acta2, foxc1b, and pdgfr $\beta$ label distinct and overlapping subsets of vascular mural cells in the developing embryo

Reliable markers to trace the development of the first vascular mural cells colonizing vessels are lacking. We therefore tested markers that are expressed in neural crest for whether they were also expressed in vascular smooth muscle around the ventral aorta, the first site to develop smooth muscle in the embryo. Such a marker would span the development of smooth muscle from when neural crest cells first attach to endothelium, to differentiation. foxc1b, as detected by the foxc1b:EGFP transgenic line, was one candidate. We first verified the transgenic line faithfully mimics endogenous foxc $1 b$ expression in mural cells. Comparing foxc $1 b$ mRNA expression at 2 and $4 \mathrm{dpf}$, patterns between the endogenous gene and transgenic line are very similar (Fig. 1A, C, E, G, I, and K). Specifically, foxc1b is expressed in the region of the aortic arches, jaw, ceratohyal (Schilling et al., 1996; Knight, 2003; Filipek-Górniok et al., 2015; Xu et al., 2018) and in the periocular mesenchyme in the ventral head. Furthermore, foxc1b mRNA expression overlaps with foxc1b:EGFP transgenic expression in the ventral aorta, aortic arch arteries, and ceratohyal at 2 and $4 \mathrm{dpf}$, suggesting EGFP in the transgenic fish line mimics endogenous mRNA expression (Fig. 1B, D, F, H, J, and L).

As Foxc1 is expressed in mural cells of mice (Siegenthaler et al., 2013), and foxc1b is expressed in endothelial cells of the early zebrafish trunk (Skarie and Link, 2009; Chen et al., 2017), we next tested the cell-type specificity of foxc1b:EGFP expression later, at $4 \mathrm{dpf}$, when robust expression of smooth muscle and pericyte markers are present in zebrafish embryos (Santoro, Pesce and Stainier, 2009; Seiler, Abrams and Pack, 2010; Wang et al., 2014; Whitesell et al., 2014). foxc1b:EGFP positive cells are located adjacent and in close contact with the endothelium of the ventral aorta (Fig. 2A, arrowheads) similar to cells expressing the classic smooth muscle marker, acta2:EGFP (Fig. 2B). We show that foxc1b:EGFP and acta2:mCherry are co-expressed in smooth muscle cells (Fig. 2C, arrows, arrowheads point to mural cells expressing only one marker). Similarly, when we compare foxc1b mRNA expression to kdrl:EGFP or acta2:EGFP 
transgenic fish we see foxc1b mRNA expressing cells are close to the endothelium ( $k d r l)$ and overlap with smooth muscle (acta2), but not the endothelium (Fig. S1). To test if foxc1b:EGFP also labels pericytes, foxc1b:EGFP was crossed with a pdgfr :Gal4FF; UAS:NTR-mCherry line (hereafter pdgfrß:mCherry). At $4 \mathrm{dpf}$, we observed no co-expression of foxc1b:EGFP and pdgfr $\beta$ :mCherry in the ventral head (Fig. 2D). pdgfr $\beta$ :mCherry expressing cells are pericytes and are located adjacent to $k d r l: E G F P$ expressing endothelial cells, and only rarely co-express acta2:EGFP (Fig. 2E, F).

foxc1b is expressed in mesenchymal cells as they associate with, and attach to, the endothelium

The earliest stages of migration and attachment of mural cells to the endothelial wall have not been observed in any species. We hypothesized that the early expression of foxc1b in neural crest and smooth muscle would allow us to visualize this process. At $2 \mathrm{dpf}$, when we have previously seen vascular mural cells present near the endothelium of the dorsal aorta using transmission electron microscopy (Liu et al., 2007; Lamont et al., 2010), foxc1b:EGFP positive cells are near the endothelium and have a mesenchymal morphology (Fig. 3A). In comparison, the acta2:mCherry transgene is not expressed in this region at this time (Fig. 3B) (Whitesell et al., 2014). By $3 \mathrm{dpf}$, foxc1b:EGFP positive cells associate closely with the endothelium (Fig. 3C, arrowheads) and some cells co-express acta2:mCherry (Fig. 3D, arrows). There are also foxc1b:EGFP positive cells that do not express acta2:mCherry. At $4 \mathrm{dpf}$, most mural cells coexpress foxc1b:EGFP and acta2:mCherry. We note that when vascular expression of acta2:mCherry begins around $3 \mathrm{dpf}$ in a few scattered cells, it is co-expressed with foxc1b:EGFP (Fig. 3D). Taken together, this suggests that foxc1b expression precedes acta2 expression in mural cells (Fig. 3C, D). foxc1b also labels smooth muscle cells at later stages. At $4 \mathrm{dpf}$, foxc1b:EGFP is still present in mural cells around the endothelium (Fig. 3E), and these cells coexpress acta2:mCherry (Fig. 3F). These patterns persist through 9 and $12 \mathrm{dpf}$ (Fig. S2) and into adulthood. There is also co-expression of foxc $1 b$ and acta 2 in the trunk, along the dorsal aorta at $4 \mathrm{dpf}$, but no endothelial expression (Fig. S3). Co-expression is not universal, and cells expressing one or other marker (foxc1b:EGFP or acta2:mCherry) are occasionally observed (Fig. S2).

To trace when foxc1b:EGFP expressing cells transform from a mesenchymal to a smooth muscle morphology we used time-lapse confocal microscopy. foxc1b:EGFP positive cells begin as mesenchymal clusters (Fig. 4A), adjacent to kdrl:mCherry positive endothelium. Distinct foxc1b:EGFP positive vascular mural cells emerge on the endothelium by $58 \mathrm{hpf}$ (Fig. 4A, Movie 1). After taking on a mural cell morphology, foxc1b:EGFP positive cells then express acta2. This 
transition from foxc1b:EGFP+; acta2:mCherry to foxc1b:EGFP+; acta2:mCherry+ cells occurs around 66 hpf (Fig. 4B, Movie 2). Taking time-lapse and marker expression together, our data depict the developmental progression for mural cells around the ventral aorta, where foxc $1 b$ expressing mesenchymal cells closely associate with the endothelium, morphologically differentiate into vascular mural cells and express the smooth muscle marker acta2.

\section{foxc1b is expressed in smooth muscle cells, but not pericytes, in the adult zebrafish brain}

Foxc1 marks both pericytes and smooth muscle cells in the mouse brain, but it is unknown whether it shows cell-type specificity of expression in zebrafish at adult stages. We find that embryonic co-expression patterns of foxc $1 \mathrm{~b}$, acta 2 and pdgfr $\beta$ at $4 \mathrm{dpf}$ are consistent in the adult brain, but also show some important differences. In the adult brain, we find that foxc1b:EGFP expressing cells are associated with large arterioles, but not smaller capillaries (Fig. 5A), similar to acta2:EGFP expressing cells (Fig. 5B). foxc1b:EGFP is almost always co-expressed with acta2:mCherry; however, there are regions of acta2:mCherry expression that lack co-expression of foxc1b:EGFP (Fig. 5C), particularly on the largest diameter vessels. We never detected foxc1b:EGFP expressing cells on the same vessels that express pdgfrß:mCherry, and foxc1b:EGFP is not expressed in endothelial cells (Fig. 5A). Thus, in both the ventral head of the embryo and the adult brain, foxc1b is expressed in smooth muscle cells, but not pericytes or endothelial cells.

\section{Mural cell marker expression changes with vessel diameter and location}

As an animal grows, its vessels enlarge to increase the supply of blood to growing tissues. We expected that mural cell types would correlate with vessel diameter, but that there might also be some variation in mural cell type depending on vessel location and developmental timing. We measured vessel diameter in vessels expressing $k d r l$ (no mural cells), pdgfr $\beta$, foxc $1 b$ or acta2 in the ventral head (ventral aorta, opercular artery, hypobranchial artery, aortic arch arteries, and lateral dorsal aorta), embryonic brain (central arteries, primordial hindbrain channel, basilar artery, and the internal carotid artery), and adult brain.

Starting at $4 \mathrm{dpf}$, when acta2 is first expressed in smooth muscle cells of the ventral aorta and associated vessels, we find that naked vessels (labelled by endothelial $k d r l$, but unlabelled with mural cell markers) have a mean diameter of $9.7 \pm 2.0 \mu \mathrm{m}$ (Fig. 6A, B). The vessels covered with pericytes (pdgfr $\beta$ ) have a mean vessel diameter of $10.3 \pm 2.1$, but are not significantly different in size from naked vessels. We find the mean vessel diameter for foxc1 $b$ covered vessels is $14.6 \pm$ $4.4 \mu \mathrm{m}$, while for acta2 covered vessels, the mean is $14.1 \pm 4.5 \mu \mathrm{m}$, and for foxc1b + acta2 
255

256

257

258

259

260

261

262

263

264

265

266

267

268

269

270

271

272

273

274

275

276

277

278

279

280

281

282

283

284

285

286

covered vessels the mean diameter is $20.5 \pm 6.8 \mu \mathrm{m}$. Thus, vessels with foxc $1 \mathrm{~b}$ or acta2 single positive mural cells are significantly larger than naked $k d r l$ or $p d g f r \beta$ covered capillaries, as one might expect for vessels covered by smooth muscle, and foxc $1 b+a c t a 2$ double marked vessels are significantly larger. The reason for larger double positive vessels is unclear, but may reflect a strong contribution of foxc $1 b$ positive cells to the ventral aorta where acta2 is heavily expressed in early development.

The embryonic brain develops smooth muscle coverage later than vessels in the ventral head, most likely as the blood flow in the brain is lower in early development. Interestingly, we found no expression of foxc $1 b$ around vessels in the $4 \mathrm{dpf}$ zebrafish brain. The mean vessel diameter for naked $(k d r l)$ brain vessels at $4 \mathrm{dpf}$ is $7.5 \pm 2.0 \mu \mathrm{m}$ while it is $8.1 \pm 2.2 \mu \mathrm{m}$ for pdgfr $\beta$ covered vessels (Fig. 6C, D). Both naked $k d r l$ and $p d g f r \beta$ covered vessel diameters are significantly smaller than acta2 covered vessels $(14.4 \pm 2.5 \mu \mathrm{m})$.

In the adult brain, the mean vessel diameter for naked vessels is $5.7 \pm 2.0 \mu \mathrm{m}$ and for $p d g f r \beta$ covered vessels it is significantly larger at $9.4 \pm 5.2 \mu \mathrm{m}$. The mean vessel diameter of foxc $1 \mathrm{~b}$ covered vessels is $17.2 \pm 11.2 \mu \mathrm{m}$, and for acta2 covered vessels the diameter is $19.9 \pm 12.5 \mu \mathrm{m}$. For vessels that express both foxc1b + acta2, the diameter is $18.8 \pm 12.0 \mu \mathrm{m}$ (Fig. 6E, F). Overall, vessels covered by foxc1b are significantly larger than both naked and $p d g f r \beta$ covered vessels, but all smooth muscle covered vessels (foxc1b, acta2, foxc1b + acta2) are of a similar size. Representative vessel sizes are depicted, to scale in Figure 6F. Thus we show that foxc1b:EGFP labels smooth muscle cells on medium and large diameter vessels in the adult brain.

\section{Mural cell populations have unique transcriptome profiles}

To probe the transcriptome of vascular smooth muscle cells in early development, we required a specific marker; however, expression of the acta2:EGFP transgene is not restricted to vascular smooth muscle cells, limiting its utility for transcriptome profiling. Thus, the intersection of foxc $1 b$ and acta2 transgene expression might provide the possibility for more selective smooth muscle cell isolation. To investigate this possibility, we isolated acta2:EGFP positive or foxc1b:EGFP + acta2:mCherry double positive cells, along with respective negative populations, and subjected them to RNA-Seq. In parallel, we also analyzed kdrl:HRAS-mCherry positive and negative cells for comparison. Consistent with a mural cell identity, both acta2:EGFP positive and foxc1b:EGFP + acta2:mCherry double positive cells show significant enriched expression of known vascular smooth muscle genes, such as acta2, tagln and fbln5, when compared to transgene negative populations (Fig. 8A, B). By contrast, neither population showed an enrichment for endothelial 
markers (cdh5, pecam1, egfl7; Fig. 8A-C). Interestingly, myocardin (myoc), a known transcriptional regulator of smooth muscle cell differentiation in mammals (Du et al., 2003) only showed significant enrichment when comparing foxc1b:EGFP + acta2:mCherry double positive cells versus negative (Fig. 8A, B), suggesting that intersectional transgene labeling improved detection of known mural cell genes. Indeed, multiple vascular smooth muscle cell expressed genes showed much higher levels of enrichment in foxc1b:EGFP + acta2:mCherry double positive cells when compared to negative cells, than acta2:egfp-positive (Fig. 8D). As noted above, all but one of these genes failed to show any enrichment in endothelial cells (Fig. 8D). To further investigate the utility of intersectional labeling of mural cell populations using foxc1b:EGFP and acta2:mCherry, we performed whole mount in situ hybridization to validate candidate genes' expression patterns. For this purpose, we chose 11 candidate transcripts whose expression was enriched in foxc1b:EGFP + acta2:mCherry double positive cells (Fig. 8E, Fig. 9). In all cases, we observed expression in putative mural cells surrounding the endothelial lining of blood vessels. Moreover, two candidate genes (capn3b and cracr2b/si:ch211-247i17.1) only displayed enrichment in foxc1b:EGFP + acta2:mCherry double positive cells, and exhibit expression in putative mural cells. Together, these observations demonstrate the potential utility for using an intersectional approach to label vascular mural cell populations. Moreover, this approach aids in the identification of potentially new candidate genes that may play a role in mural cell development or function.

\section{foxc1 genes are required for smooth muscle development}

As foxc1b is expressed in cells that differentiate into acta2 positive smooth muscle cells, we next tested whether foxc1 $\mathrm{b}$ and its paralog foxc1a are necessary for smooth muscle development. The two genes have similar expression patterns, and foxc1a and foxc1b can compensate for each other (Jolanta M. Topczewska et al., 2001; Skarie and Link, 2009; Xu et al., 2018). foxc1a is highly expressed in our acta2 dataset, thus we scored for acta2:EGFP positive smooth muscle cell coverage of the ventral aorta in foxc1a and foxc1b compound mutants. Incrosses of foxc1 $\mathrm{a}^{+/ \text {; }}$ foxc $1 b^{+/-}$compound heterozygote zebrafish provided all 9 genotype combinations (Fig. 10A-I). In addition to the mutant background, all embryos were heterozygous for the acta2:EGFP transgene to visualize smooth muscle. Wild type foxc $1 \mathrm{a}^{+/+} ;$foxc $1 b^{+/+}$embryos have a mean length of smooth muscle coverage of $(102.3 \mu \mathrm{m})$. Loss of foxc1b alleles in a foxc1 $\mathrm{a}^{+/+}$background causes no significant change in coverage $\left(\right.$ foxc $1 \mathrm{a}^{+/+} ;$foxc $1 b^{+/-}=134.7 \mu \mathrm{m}$, foxc $1 \mathrm{a}^{+/+}$; foxc $1 b^{-/-}=94.0 \mu \mathrm{m}$, Fig. $10 \mathrm{~A}-\mathrm{C})$. There is also no significant change with heterozygous loss of $\mathrm{foxc} \mathrm{a}^{+/-}\left(\right.$foxc $1 \mathrm{a}^{+/-}$; foxc $1 b^{+/+}=99.5 \mu \mathrm{m}$, foxc $1 a^{+/-}$; foxc $1 b^{+/-}=101.9 \mu \mathrm{m}$, and foxc1 $a^{+/-;}$foxc $1 b^{-/-}=83.4 \mu \mathrm{m}$; Fig. 10D - 
320 F). However, homozygous loss of foxc1 $\mathrm{a}^{-/}$has a strong effect on smooth muscle coverage when

321 combined with loss of foxc $1 b$. foxc $1 a^{-/-;}$foxc $1 b^{+/+}$embryos are not significantly different from wild

322 type $(94.1 \mu \mathrm{m})$. In contrast, foxc $1 \mathrm{a}^{-/-;}$; foxc $1 \mathrm{~b}^{+/-}(3.2 \mu \mathrm{m})$, and foxc $1 \mathrm{a}^{-/-}$; foxc $1 \mathrm{~b}^{-/}(13.1 \mu \mathrm{m}$; Fig. 10G

$323-$ I) embryos have severely reduced smooth muscle coverage on the ventral aorta. Thus, the

324 length of smooth muscle coverage along the ventral aorta decreases with increased loss of foxc1

325 alleles, with loss of 3 or 4 alleles having the most significant reduction in the length of smooth

326 muscle coverage (Fig. 10J).

327 Of note, when both alleles of foxc1a are lost, these embryos initially develop circulation but 328 present with progressive cardiac edema and circulatory insufficiency around 72 hpf (Fig. S5).

329 These data suggest that not only is foxc1 expressed early in the smooth muscle lineage, but that 330 foxc1 ( $a$ and $b$ ) are critical for the early development of smooth muscle cells, and cardiac function.

331 Homozygous loss of foxc1a coupled with loss of one or two alleles of foxc1b has the strongest 332 effect on vascular development, but the converse is not true. Loss of two alleles of foxc $1 b$ and one allele of foxc1a has a wild type phenotype.

334 Discussion:

335 Vascular mural cells are required for maintenance, contraction, and support of the underlying 336 endothelium; however, we still have limited tools to study their early development, in vivo. Current 337 common markers of smooth muscle cells (acta2, tagln) and pericytes ( $p d g f r \beta, n g 2)$ have relatively 338 specific and robust expression in the cells of interest but smooth muscle markers are not 339 expressed early enough in development in the zebrafish model to study early mural cell 340 differentiation. Furthermore, neural crest markers such as sox10 turn off before smooth muscle 341 cells differentiate in fish, unless utilizing lineage tracing techniques (Cavanaugh, Huang and 342 Chen, 2015). However, we know that neural crest cells are precursors of vascular smooth muscle 343 and pericytes in zebrafish (Cavanaugh, Huang and Chen, 2015; Ando et al., 2016; Stratman et 344 al., 2017), and we can identify vascular mural cells using transmission electron microscopy before 345 we observe expression of smooth muscle markers (Liu et al., 2007; Lamont et al., 2010). 346 Identification of additional markers is therefore necessary to observe these intermediate 347 differentiation states. We show here foxc1b:EGFP labels early mesenchymal cells and smooth 348 muscle cells in vivo and we can observe these cells undergoing morphological changes from a 349 mesenchymal to a smooth muscle morphology. We use foxc1b to study morphological 350 differentiation of smooth muscle cells in real time, the interplay between mural cell marker 351 expression, vessel size and its transcriptome, as well as the role of foxc1 in smooth muscle 
differentiation. Furthermore, selecting a foxc1b + acta2 positive subset of mural cells identifies an enriched vascular mural cell population.

\section{foxc1b is involved in early vascular smooth muscle cell differentiation}

We first established that foxc1b expressing cells co-express a smooth muscle marker, acta2, as in mouse (Kume et al., 2001; Siegenthaler et al., 2013). Unlike mouse, foxc1b:EGFP is not coexpressed in head endothelial cells or pericytes, suggesting that some foxc1b:EGFP expressing cells are, or will become smooth muscle cells in zebrafish. Three lines of evidence suggest that foxc $1 b$ is expressed in mesenchymal precursors that will become acta2 positive smooth muscle cells. First, we observe mesenchymal expression of foxc $1 \mathrm{~b}$ :EGFP near vessels at $2 \mathrm{dpf}$ and show how these cells undergo morphogenesis as they adhere to endothelium and adopt a smooth muscle morphology. Secondly, foxc1b is expressed in vascular mural cells before the earliest acta2 expression, and at later timepoints, the two genes are strongly co-expressed. Thirdly, loss of foxc1 leads to a strong reduction in the number and the coverage by acta2 positive cells on the ventral aorta. Taken together, these data suggest foxc1b expressing mesenchymal precursors differentiate into acta2 expressing vascular smooth muscle cells on the ventral aorta.

In mice, FOXC proteins are regulated by Sonic hedgehog (Shh), a signaling pathway driving mural cell differentiation (Yamagishi et al., 2003; Lamont et al., 2010). Given that foxc1b is expressed in neural crest (Seo and Kume, 2006; Inman et al., 2013; Koo and Kume, 2013; Seo et al., 2017), that lineage tracing of sox10-expressing neural crest cells show they contribute to smooth muscle on the bulbus arteriosus and ventral aorta (Mongera et al., 2013; Cavanaugh, Huang and Chen, 2015), and FOXC proteins are expressed in mural cells in mouse and fish (Kume et al., 2001; Siegenthaler et al., 2013; French et al., 2014), our data are consistent with previous models of the origin of the first vascular smooth muscle cells in the head of the embryo (Etchevers et al., 2001; Calloni et al., 2007; Mongera et al., 2013; Wang et al., 2014; Cavanaugh, Huang and Chen, 2015).

The role of foxc1 in smooth muscle differentiation in zebrafish is unique. For instance, compound Foxc1 and Foxc2 mutant mouse vessels maintain acta2 positive smooth muscle coverage, although vessel morphology is altered, with only enlarged vessels present (Kume et al., 2001). However, in fish we show that the loss of at least 3 alleles of foxc $1 \mathrm{a} / \mathrm{b}$ prevents acta2 positive smooth muscle from differentiating along the ventral aorta. Similar to the morpholino knockdown of foxc1a and foxc1b (French et al., 2014), loss of both paralogs is required for significant loss of smooth muscle coverage, with foxc1a having a stronger effect on smooth muscle differentiation 
than foxc1b. There is known genetic redundancy in zebrafish foxc1 genes (Jolanta M. Topczewska et al., 2001; Xu et al., 2018). foxc1a has a similar expression pattern, to foxc1b (Jolanta M. Topczewska et al., 2001; Xu et al., 2018) and Fox family members are known to play redundant roles in mice (Kume et al., 2001; Seo and Kume, 2006).

\section{Localization of mural cell subsets and the transcriptomic differences among embryonic} mural cells

foxc1 is expressed in multiple cell types in both the brain and trunk of mice and zebrafish. In the trunk of the zebrafish, foxc1b mRNA is expressed within endothelial cells during very early angiogenesis (Skarie and Link, 2009; Chen et al., 2017); however, we do not see transgenic foxc1b:EGPF expression in head or trunk endothelial cells at the late developmental stages we examine (4 dpf through adult) (Kume et al., 1998; Prasitsak et al., 2015). Surprisingly, despite reported strong expression of Foxc1 in mouse brain pericytes, we do not see foxc1b expression in fish brain pericytes at any stage (Zarbalis et al., 2007; Siegenthaler et al., 2013; Mishra et al., 2016). Instead we find that foxc1b:EGFP expression is within smooth muscle cells, with a similar expression pattern to acta2. Furthermore, we show that foxc1a and $b$ expression is required at an early stage in the differentiation of acta2 positive smooth muscle cells around the ventral aorta. These differences in expression may reflect species or developmental staging differences between fish and mammals, or incomplete elements in the enhancer used to generate the foxc $1 \mathrm{~b}$ transgene.

We wanted to understand whether foxc1b and acta2 are always co-expressed and what type of vessels have mural cells expressing these markers. We chose to examine this by vessel diameter, a measurement that is relatively constant for capillaries across species. The smallest capillary vessel diameters are set by the size of the erythrocyte (about 7-8 $\mu \mathrm{m}$ in diameter in humans and zebrafish) (Kulkeaw and Sugiyama, 2012) and are therefore comparable cross-species (Hartmann et al., 2015). We found a complete lack of co-expression of foxc1b with pdgfr $\beta$, a pericyte marker and therefore absence of foxc1b expressing cells from the smallest vessels of the vascular system. Instead, we found a hierarchical continuum of mural cell marker expression, with smooth muscle marked by foxc1b and acta2 on larger diameter vessels. As the ventral aorta is the largest vessel in the embryonic ventral head, it is subject to larger blood pressure changes and needs the largest amount of smooth muscle coverage in the early developing embryo $(\mathrm{Hu}$, Joseph Yost and Clark, 2001; Whitesell et al., 2014). Therefore, the need for more mature, contractile smooth muscle early in development as compared to the brain, may require early foxc1b and acta2 co-expression. These results are consistent with other reports in mouse 
417 (Hartmann et al., 2015; Grant et al., 2017), such as the recently observed zonation of mural cells

418 in the mouse brain (Vanlandewijck et al., 2018).

\section{Transcriptomic differences among embryonic mural cells}

420 We used a combination of two markers, acta2 (vascular and visceral smooth muscle, in addition 421 to cardiac and some skeletal muscle cells (Whitesell et al., 2014)) and foxc1b (vascular smooth 422 muscle cells but also pharyngeal arch, ceratohyal, and migratory cranial mesenchymal cells in 423 zebrafish (Jolanta M. Topczewska et al., 2001; Miesfeld and Link, 2014)) for FACS to isolate 424 vascular mural cells. This restricts our population to an enriched vascular smooth muscle 425 population. Analyzing the results of the RNA-Seq experiment, it appears that using a double 426 sorted population for both foxc1b and acta2 is a powerful strategy for identifying and isolating 427 vascular smooth muscle cells, as each marker alone is expressed in other cell types, but the 428 intersection of the two markers marks a strongly enriched population of vascular smooth muscle 429 cells.

430 Within the transcriptomes, we observe strong expression of expected genes such as acta2, tagln, 431 tpm4b, and desmb, all of which show enriched vascular or visceral smooth muscle expression by 432 in situ hybridization. Other muscle related genes (for example, myosins, ion channels, and 433 receptors) are present, and we also identify many genes which are novel, denoted only by 434 chromosomal location that should be investigated as potential mural cell markers. A few genes we tested were not expressed in mural cells; however, we found that most were expressed near blood vessels (data not shown), suggesting the enzymatic dissociation prior to sorting may not have created single cell suspensions (Vanlandewijck et al., 2018). Inclusion of a comparison to an endothelial transcriptome (kdrl:HRAS-mCherry) reduced the potential for endothelial contamination in the dataset that may arise from incomplete dissociation of cells prior to sorting.

Our smooth muscle cell transcriptomes are not only the first zebrafish mural cell transcriptomes, but also one of the few to profile mural cells in early development. We sorted zebrafish cells at 4

$442 \mathrm{dpf}$, near the onset of smooth muscle marker expression, whereas most mouse transcriptomic 443 studies used postnatal or adult mice (Lee et al., 2015; He et al., 2016; Vanlandewijck et al., 2018). 444 One study using pdgfb and pdgfr $\beta$ mutant mice at E17.5 (Bondjers et al., 2006), was also 445 embryonic, but is at a stage multiple days after the onset of classic mural cell marker expression 446 in embryonic mice (Lindahl et al., 1997; Hellström et al., 1999). In postnatal mice, pdgfr $\beta$ and $n g 2$ 447 double positive pericytes have been sequenced (He et al., 2016) and compared to four other 448 pericyte studies, resulting in a pooled brain mural cell transcriptome. The transcriptomes of mouse 
visceral smooth muscle(Lee et al., 2015), and single cell transcriptomes of the brain vasculature to identify endothelial, smooth muscle, pericyte, fibroblast, and astrocyte cells (Vanlandewijck et al., 2018) are complementary to our data.

452 Comparing our smooth muscle transcriptomes (acta2, foxc1b + acta2) to the previously published mouse smooth muscle transcriptome datasets (Lee et al., 2015; Vanlandewijck et al., 2018), we

454 find many genes shared between the datasets, as expected. These include the common mural cell markers acta2, tagln, desmb, cnn1b, myl9a, and mylh11a that are also observed in colonic and jejunal smooth muscle transcriptomes (Lee et al., 2015), and arterial, arteriolar, or venous smooth muscle cell transcriptomes (Vanlandewijck et al., 2018). As our acta2:EGFP transcriptome will contain transcripts from vascular and visceral smooth muscle, it is expected that we find many common genes. However, foxc1b:EGFP + acta2:mCherry represents an enriched vascular smooth muscle cell transcriptome, making it unique. There are 303 genes shared between the acta2 and foxc1b + acta2 datasets, and 1644 genes specific to the foxc $1 b+$ acta2 dataset. These include several hundred novel genes that will be interesting to pursue. Common smooth muscle genes are present, including Imod1, fh/2a, elnb, mylh11a, myocd, and kcne 4 and have different abundances in the acta2 and foxc $1 b+$ acta2 datasets. For example, Imod1 is expressed in both datasets, but is more abundant in the acta2 dataset, and by in situ hybridization, Imod1 shows high expression in visceral smooth muscle. fh/2a, elnb, myhl11a, myocd, kcne4 are also found in the visceral smooth muscle transcriptomes (Lee et al., 2015), and indicate common smooth muscle genes. In addition, there are $\sim 40$ uncharacterized novel genes 469 specific to both the acta2 and foxc1b + acta2 datasets, which warrant further investigation as 470 potential smooth muscle specific genes.

471 In conclusion, we have utilized foxc1b expression to identify a window where mesenchymal cells 472 undergo a morphological change to become early smooth muscle cells associated with the 473 endothelium in real-time. This transition requires foxc $1 b$ for the mesenchymal cells to differentiate 474 into mature, acta2 expressing smooth muscle cells. In mature smooth muscle, foxc1b marks a 475 unique type of smooth muscle, a subset of acta2 expressing smooth muscle on medium diameter 476 vessels. RNA-Seq data show key gene expression signatures of different subsets of vascular 477 smooth muscle cells. These data will allow for a better understanding of how early smooth muscle cells behave, function, and undergo differentiation. 


\section{Materials and Methods:}

480 Complete methods are available in the supplementary methods.

\section{Ethics, husbandry, and strains:}

482

Zebrafish husbandry was performed following standard protocols (Westerfield, 1995). All procedures were approved by the University of Calgary Animal Care Committee, the University of Massachusetts Medical School IACUC, and the University of Alberta Animal Care and Use type Tupfel long fin (TL), Tg(kdrl:mCherry) ${ }^{c i 5}$ (Proulx, Lu and Sumanas, 2010), Tg(kdrl:EGFP)la116 al., 2014), Tg(acta2:mCherry) ${ }^{\text {cas }}$ (Whitesell et al., 2014), TgBAC(pdgfrß:Gal4FFca42 (Ando et al., 2016); UAS-NTR:mCherryc264 (Davison et al., 2007)), hereafter Tg(pdgfrß:mCherry), Tg(5.0kbfoxc1b:EGFP)mw44 (Miesfeld and Link, 2014).

\section{Generation of foxc1a and foxc1b mutant strains:}

492 Generation of foxc1 $1 \mathrm{a}^{\text {ua1017 }}$ and foxc1bua1018 CRISPRC mutant strains each required two guide RNAs, produced by cloning into pDR274 (Addgene, \#42250), followed by in vitro transcription using the MAXIscript T7 Transcription kit (Ambion, Cat. No. AM1312). foxc1aua1017 target mMACHINE T7 Transcription kit (Ambion, Cat. No. AM1344), followed by a Poly-A Tailing Kit (Ambion, AM1350). Injections were standardized using $30 \mathrm{ng} / \mu \mathrm{l}$ per gRNA and $300 \mathrm{ng} / \mu \mathrm{l}$ Cas9. Injected fish (P0) were outcrossed and embryos were screened using HRM (Qiagen, Rotor-Gene Q). Mutations were cloned and sequenced from genomic DNA and RNA.

\section{Sectioning, imaging and image analysis:}

504 Confocal images were collected on a Zeiss LSM 700 microscope. Embryos were mounted in 0.8\% 505 low melt agarose on glass bottom dishes (MatTek, Ashland, MA, Cat. No. P50G-0-30-F), and 506 were incubated in a heated chamber with the addition of $0.4 \%$ Tricaine (Sigma, Cat. No. A5040)

507 for restraint. Representative images are shown.

508 Adult zebrafish brains (0.5 - 1.5 years old) were dissected and fixed overnight in 4\% PFA/1x PBS. 509 Brains were washed in PBS (3x $5 \mathrm{~min})$ before being mounted in a $55^{\circ} \mathrm{C}$ gelatin solution $(15 \%$ 
w/v). Coronal vibratome sections were cut on a Leica Vibratome VT1000S at a thickness between $150-300 \mu \mathrm{m}$.

Wholemount imaging of stained samples was conducted with a Zeiss Stemi SV11 microscope, with a Zeiss HR camera. Stained wholemount embryos were sectioned at $8 \mu \mathrm{m}$ after mounting in JB4 resin (Polysciences, Warrington, PA). Sections were imaged on a Zeiss Axio Imager.Z1 microscope with an AxioCam ICc5 camera (Zeiss).

Vessel diameter measurements were measured from confocal images using FIJI/ImageJ (Schindelin et al., 2012). Diameters were measured from the external diameter of the endothelium, away from nuclei of mural and endothelial cells. Seven measurements were taken for each sample where possible. Ventral head measurements were taken from the ventral aorta and the aortic arch arteries. In the brain, measurements were predominantly taken from the internal carotid artery (smooth muscle) and central arteries (pericytes). Measurements represent mean vessel diameter \pm standard deviation in micrometers.

\section{In situ hybridization and antibody staining:}

In situ hybridization was performed using the method of Lauter et al. 2011 (Lauter, Soll and Hauptmann, 2011), with the following modifications. Embryos were permeabilized in 2\% $\mathrm{H}_{2} \mathrm{O}_{2}$ /methanol for 20 minutes and depigmented in $3 \% \mathrm{H}_{2} \mathrm{O}_{2} / 0.5 \% \mathrm{KOH}$ in water for 10 minutes. Proteinase $\mathrm{K}$ permeabilization was at $10 \mu \mathrm{g} / \mathrm{ml}$ for 1 hour for $4 \mathrm{dpf}$ embryos. EGFP was detected with 1:500 mouse aGFP (JL-8, BD Clontech/Takara Bio USA) and the Vectastain ABC Kit (Vector Laboratories, Burlingame CA, USA) using 1:400 aDIG POD Fab fragment (Roche, Cat. No. 11 207733 910) and FAM (ThermoFisher, Cat. No. C1311) for green fluorescent probe signal, or mouse aGFP (1:500, JL-8, BD Clontech/Takara Bio USA) and Alexa555 goat a mouse fluorescent secondary antibody (1:500, ThermoFisher, Cat. No. A21422). Primers for in situ hybridization are listed in Supplemental Table 3.

\section{FACS \& RNA isolation:}

The protocol for single cell dissociation was based upon Rougeot et al. 2014. 300 acta2:EGFP and 300 foxc1b:EGFP + acta2:mCherry (4 dpf) fish were anesthetized with $0.4 \%$ Tricaine (Sigma) and pooled. Embryos were treated for 15 minutes with calcium-free Ringers Solution and triturated 15 times. Dissociation Solution was added and triturated 10 times before placing in a $28.5^{\circ} \mathrm{C}$ water bath with shaking at $60 \mathrm{rpm}$ and periodic trituration for 1 hour. The reaction was stopped, centrifuged and resuspended in Dulbecco's Phosphate-Buffered Saline (GIBCO by Life 
541 Technologies; REF 14040-133), centrifuged and resuspended in fresh Resuspension Solution.

542 The single cell suspension was filtered with $75 \mu \mathrm{m}$, followed by $35 \mu \mathrm{m}$ filters. Cells were then

543 sorted with a BD FACSAria III (BD Bioscience, San Jose, USA) and collected into a collection

544 solution. RNA Isolation of sorted cells was performed using the Trizol method (Ambion by Life

545 Technologies; Carslbad CA, USA; Cat No. 15596026). For analysis of kdrl:HRAS-mCherry cells,

546 larvae bearing transgene were anesthetized at $5 \mathrm{dpf}$, dissociated into single cell suspensions,

547 fixed, and subjected to fluorescence activated cell sorting (FACS) by the University of

548 Massachusetts Medical School Flow Cytometry Core, as described previously (Quillien et al.,

549 2017).

550 Next Generation Sequencing and bioinformatics:

551 Library preparations used the REPLI-g Single Cell Kit (Cat. No. 150343, Qiagen) for paired end

552 sequencing of $4 \mathrm{dpf}$ samples, using an Illumina NextSeq Platform. $4 \mathrm{dpf}$ samples were:

553 acta2:EGFP , acta2:EGFP; and foxc1b:EGFP+ + acta2:mCherry ${ }^{+}$, foxc1b:EGFP +

554 acta2:mCherry). RNA isolation and library preparation for kdrl:HRAS-mCherry positive and

555 negative cell populations was handled as previously reported (Quillien et al., 2017).

556 The sequencing depth for acta2:EGFP positive samples averaged 54.8 million reads and 60.9

557 million reads for the negative population. The sequencing depth for foxc $1 b: E G F P+$

558 acta2:mCherry double positive samples averaged 59.7 million reads and 61.5 million reads for

559 the double negative population. The sequencing depth for kdrl:HRAS-mCherry samples

560 averaged 52.7 million reads for the positive population and 39.1 million reads for the negative

561 population.

562 Paired-end reads were aligned to 26 chromosomes and 967 primary assembly scaffolds of the

563 zebrafish genome GRCz11, with star_2.5.3a (Dobin et al., 2013). Aligned exon fragments with

564 mapping quality higher than 20 were counted toward gene expression with featureCounts_1.5.2

565 (Liao, Smyth and Shi, 2014). Normalization and differential expression (DE) analysis was

566 performed with DESeq2_1.20.0 (Love, Huber and Anders, 2014). For DE analysis, the original

567 DESeq2 shrinkage estimator was used to estimate log2 fold change (LFC) for each comparison.

568 False discovery rate $($ FDR $<0.05)$ and $|L F C|>1$ was used as a cut-off to identify significantly

569 enriched genes. Parameters: GTF: GCF_000002035.6_GRCz11_genomic.ucsc.primary.gff.

570 Genome: danRer11.primary.fa

571 Smooth muscle quantification 
572 Heterozygote transgenic acta2:EGFP fish were imaged, and then FIJI/ImageJ was used to

573 analyze the length of smooth muscle coverage upon the ventral aorta (Schindelin et al., 2012).

574 Measurements were recorded from where the bulbus arteriosus merges with the ventral aorta to

575 the distal tip of smooth muscle expression on the ventral aorta or to the bifurcation point of the

576 ventral aorta, whichever was shorter (Isogai, Horiguchi and Weinstein, 2001).

577 Acknowledgments:

578 We would like to thank Charlene Watterston, Jasper Greysson-Wong, Nabila Bahrami, and

579 Danielle Blackwell, in addition to the laboratory of Dr. Peng Huang for their helpful comments on

580 the project and paper. We would like to thank the members of the Flow Cytometry core facility

581 and the Alberta Children's Hospital Research Institute (ACHRI) Genomics Platform at the

582 University of Calgary.

583 Competing interests: No competing interests declared

584 Funding: This work was funded by Natural Sciences and Engineering Research Council of 585 Canada Discovery Grant (RGPIN 06360-2014) to SJC. SJC was a Canada Research Chair Tier

586 II and an Alberta Innovates Health Solutions Senior Scholar. TRW was funded by scholarships

587 from the Alberta Innovates Health Solutions Graduate Studentship, the Kertland Family Doctoral

588 Scholarship in Vascular Biology, and an Achievers in Medical Sciences Scholarship from the

589 University of Calgary. AW was funded by Natural Sciences and Engineering Research Council

590 of Canada Discovery Grant (RGPIN 298371). OJL was funded by the Canadian Institutes of

591 Health Research (MOP-133658) and the Women and Children's Health Research Institute. NDL

592 was funded by the National Institutes of Health - National Heart, Lung and Blood Institute

593 (R35HL140017).

594 Data availability: RNA sequencing data is available from the Gene Expression Omnibus (GEO)

595 site (www.ncbi.nlm.nih.gov/geo/) with accession number GSE119718. 


\section{References:}

Abrams, J. et al. (2016) 'Graded effects of unregulated smooth muscle myosin on intestinal architecture, intestinal motility and vascular function in zebrafish', Disease Models \& Mechanisms, 9(5), pp. 529-540. doi: 10.1242/dmm.023309.

Ando, K. et al. (2016) 'Clarification of mural cell coverage of vascular endothelial cells by live imaging of zebrafish', Development, 143(8), pp. 1328-1339. doi: 10.1242/dev.132654.

Armulik, A., Genové, G. and Betsholtz, C. (2011) 'Pericytes: Developmental, Physiological, and Pathological Perspectives, Problems, and Promises', Developmental Cell, 21(2), pp. 193-215. doi: 10.1016/j.devcel.2011.07.001.

Avasarala, J. R., Jones, J. R. and Rogers, C. R. (2018) 'Forkhead box C1 gene variant causing glaucoma and small vessel angiopathy can mimic multiple sclerosis', Multiple Sclerosis and Related Disorders. Elsevier B.V., 22(February), pp. 157-160. doi: 10.1016/j.msard.2018.04.004.

Baron-Menguy, C. et al. (2017) 'Increased Notch3 Activity Mediates Pathological Changes in Structure of Cerebral Arteries', Hypertension, 69(1), pp. 60-70. doi:

\subsection{1/HYPERTENSIONAHA.116.08015.}

Bergers, G. and Song, S. (2005) 'The role of pericytes in blood-vessel formation and maintenance', Neuro-Oncology, 7(4), pp. 452-464. doi: 10.1215/S1152851705000232.

Berry, F. B. et al. (2008) 'FOXC1 is required for cell viability and resistance to oxidative stress in the eye through the transcriptional regulation of FOXO1A', Human Molecular Genetics, 17(4), pp. 490-505. doi: 10.1093/hmg/ddm326.

Berthiaume, A.-A. et al. (2018) 'Dynamic Remodeling of Pericytes In Vivo Maintains Capillary Coverage in the Adult Mouse Brain', Cell Reports, 22(1), pp. 8-16. doi:

10.1016/j.celrep.2017.12.016.

Birbrair, A. et al. (2017) 'How plastic are pericytes?', Stem Cells and Development, (55), p. scd.2017.0044. doi: 10.1089/scd.2017.0044.

Bondjers, C. et al. (2006) 'Microarray analysis of blood microvessels from PDGF-B and PDGFRbeta mutant mice identifies novel markers for brain pericytes.', The FASEB journal : official publication of the Federation of American Societies for Experimental Biology, 20(10), pp. 17031705. doi: 10.1096/fj.05-4944fje.

Calloni, G. W. et al. (2007) 'Sonic Hedgehog promotes the development of multipotent neural 
crest progenitors endowed with both mesenchymal and neural potentials', Proc Natl Acad Sci U $S$ A, 104(50), pp. 19879-19884. doi: 10.1073/pnas.0708806104.

Carmeliet, P. and Jain, R. K. (2011) 'Molecular mechanisms and clinical applications of angiogenesis', Nature, 473(7347), pp. 298-307. doi: 10.1038/nature10144.

Cavanaugh, A. M., Huang, J. and Chen, J. N. (2015) 'Two developmentally distinct populations of neural crest cells contribute to the zebrafish heart', Developmental Biology. Elsevier, 404(2), pp. 103-112. doi: 10.1016/j.ydbio.2015.06.002.

Chen, X. et al. (2017) 'Cilia Control Vascular Mural Cell Recruitment in Vertebrates', Cell Reports. ElsevierCompany., 18(4), pp. 1033-1047. doi: 10.1016/j.celrep.2016.12.044.

Chi, N. C. et al. (2008) 'Foxn4 directly regulates', Genes \& Development, (415), pp. 734-739. doi: 10.1101/gad.1629408.734.

Choi, J. et al. (2007) 'FoxH1 negatively modulates flk1 gene expression and vascular formation in zebrafish', Developmental Biology, 304(2), pp. 735-744. doi: 10.1016/j.ydbio.2007.01.023.

Craggs, L. J. L. et al. (2014) 'Microvascular pathology and morphometrics of sporadic and hereditary small vessel diseases of the brain', Brain pathology (Zurich, Switzerland), 24(5), pp. 495-509. doi: 10.1111/bpa.12177.

Craggs, L. J. L. et al. (2015) 'Immunolocalization of platelet-derived growth factor receptor-?? (PDGFR-??) and pericytes in cerebral autosomal dominant arteriopathy with subcortical infarcts and leukoencephalopathy (CADASIL)', Neuropathology and Applied Neurobiology, 41(4), pp. 557-570. doi: 10.1111/nan.12188.

Davison, J. M. et al. (2007) 'Transactivation from Gal4-VP16 transgenic insertions for tissuespecific cell labeling and ablation in zebrafish', Developmental Biology, 304(2), pp. 811-824. doi: 10.1016/j.ydbio.2007.01.033.

Dias Moura Prazeres, P. H. et al. (2017) 'Pericytes are heterogeneous in their origin within the same tissue', Developmental Biology. Elsevier Inc., (May), pp. 0-1. doi:

10.1016/j.ydbio.2017.05.001.

Van Dijk, C. G. M. et al. (2015) 'The complex mural cell: Pericyte function in health and disease', International Journal of Cardiology. Elsevier Ireland Ltd, 190(1), pp. 75-89. doi: 10.1016/j.ijcard.2015.03.258. 
Dobin, A. et al. (2013) 'STAR: Ultrafast universal RNA-seq aligner', Bioinformatics, 29(1), pp. 15-21. doi: 10.1093/bioinformatics/bts635.

Du, K. L. et al. (2003) 'Myocardin Is a Critical Serum Response Factor Cofactor in the Transcriptional Program Regulating Smooth Muscle Cell Differentiation', Molecular and Cellular Biology, 23(7), pp. 2425-2437. doi: 10.1128/MCB.23.7.2425-2437.2003.

Etchevers, H. C. et al. (2001) 'The cephalic neural crest provides pericytes and smooth muscle cells to all blood vessels of the face and forebrain.', Development (Cambridge, England), 128(7), pp. 1059-1068. doi: 10.7554/elife.10036.016.

Fatima, A. et al. (2016) 'Foxc1 and Foxc2 deletion causes abnormal lymphangiogenesis and correlates with ERK hyperactivation', Journal of Clinical Investigation, 126(7), pp. 2437-2451. doi: $10.1172 / \mathrm{JCl} 80465$.

Filipek-Górniok, B. et al. (2015) 'The NDST gene family in Zebrafish: Role of Ndst1b in pharyngeal arch formation', PLoS ONE, 10(3), pp. 1-19. doi: 10.1371/journal.pone.0119040.

French, C. R. et al. (2014) 'Mutation of FOXC1 and PITX2 induces cerebral small-vessel disease.', The Journal of clinical investigation. doi: 10.1172/JCI75109.

Gays, D. et al. (2017) 'An exclusive cellular and molecular network governs intestinal smooth muscle cell differentiation in vertebrates', Development, 144(3), pp. 464-478. doi: 10.1242/dev.133926.

Georgijevic, S. et al. (2007) 'Spatiotemporal expression of smooth muscle markers in developing zebrafish gut', Developmental Dynamics, 236(6), pp. 1623-1632. doi: 10.1002/dvdy.21165.

Grant, R. I. et al. (2017) 'Organizational hierarchy and structural diversity of microvascular pericytes in adult mouse cortex', Journal of Cerebral Blood Flow \& Metabolism, p.

0271678X1773222. doi: 10.1177/0271678X17732229.

Guimar??es-Camboa, N. et al. (2017) 'Pericytes of Multiple Organs Do Not Behave as Mesenchymal Stem Cells In??Vivo', Cell Stem Cell, 20(3), p. 345-359.e5. doi: 10.1016/j.stem.2016.12.006.

Guo, D. C. et al. (2009) 'Mutations in Smooth Muscle Alpha-Actin (ACTA2) Cause Coronary Artery Disease, Stroke, and Moyamoya Disease, Along with Thoracic Aortic Disease', American Journal of Human Genetics. The American Society of Human Genetics, 84(5), pp. 617-627. doi: 
10.1016/j.ajhg.2009.04.007.

Hartmann, D. A. et al. (2015) 'Pericyte structure and distribution in the cerebral cortex revealed by high-resolution imaging of transgenic mice', Neurophotonics, 2(4), p. 041402. doi:

10.1117/1.NPh.2.4.041402.

Hayashi, H. and Kume, T. (2008a) 'Forkhead transcription factors regulate expression of the chemokine receptor CXCR4 in endothelial cells and CXCL12-induced cell migration', Biochemical and Biophysical Research Communications, 367(3), pp. 584-589. doi: 10.1016/j.bbrc.2007.12.183.

Hayashi, H. and Kume, T. (2008b) 'Foxc transcription factors directly regulate DII4 and hey2 expression by interacting with the VEGF-notch signaling pathways in endothelial cells', PLoS ONE, 3(6), pp. 1-9. doi: 10.1371/journal.pone.0002401.

He, L. et al. (2016) 'Analysis of the brain mural cell transcriptome', Scientific Reports. Nature Publishing Group, 6(1), p. 35108. doi: 10.1038/srep35108.

Hellström, M. et al. (1999) 'Role of PDGF-B and PDGFR-beta in recruitment of vascular smooth muscle cells and pericytes during embryonic blood vessel formation in the mouse.', Development (Cambridge, England), 126(14), pp. 3047-3055. Available at: http://www.ncbi.nlm.nih.gov/pubmed/10375497.

Henshall, T. L. et al. (2015) 'Notch3 is necessary for blood vessel integrity in the central nervous system', Arteriosclerosis, Thrombosis, and Vascular Biology, 35(2), pp. 409-420. doi: 10.1161/ATVBAHA.114.304849.

Hill, R. A. et al. (2015) 'Regional Blood Flow in the Normal and Ischemic Brain Is Controlled by Arteriolar Smooth Muscle Cell Contractility and Not by Capillary Pericytes', Neuron. Elsevier Inc., 87(1), pp. 95-110. doi: 10.1016/j.neuron.2015.06.001.

Hu, N., Joseph Yost, H. and Clark, E. B. (2001) 'Cardiac morphology and blood pressure in the adult zebrafish', Anatomical Record, 264(1), pp. 1-12. doi: 10.1002/ar.1111.

Inman, K. E. et al. (2013) 'Interaction between Foxc1 and Fgf8 during Mammalian Jaw Patterning and in the Pathogenesis of Syngnathia', PLoS Genetics, 9(12). doi: 10.1371/journal.pgen.1003949.

Isogai, S., Horiguchi, M. and Weinstein, B. M. (2001) 'The Vascular Anatomy of the Developing Zebrafish: An Atlas of Embryonic and Early Larval Development', Developmental Biology, 
230(2), pp. 278-301. doi: 10.1006/dbio.2000.9995.

Ivanova, E. A. and Orekhov, A. N. (2016) 'Cellular Model of Atherogenesis Based on Pluripotent Vascular Wall Pericytes', Stem Cells International, 2016. doi: 10.1155/2016/7321404.

Jang, I. H. et al. (2015) 'Notch1 acts via Foxc2 to promote definitive hematopoiesis via effects on hemogenic endothelium', Blood, 125(9), pp. 1418-1426. doi: 10.1182/blood-2014-04568170 .

Joutel, A. et al. (1996) 'Notch3 mutations in CADASIL, a hereditary adult-onset condition causing stroke and dementia', Nature, pp. 707-710. doi: 10.1038/383707a0.

Knight, R. D. (2003) 'Lockjaw Encodes a Zebrafish Tfap2a Required for Early Neural Crest Development', Development, 130(23), pp. 5755-5768. doi: 10.1242/dev.00575.

Kodo, K. et al. (2017) 'Regulation of Sema3c and the Interaction between Cardiac Neural Crest and Second Heart Field during Outflow Tract Development', Scientific Reports. Springer US, 7(1), p. 6771. doi: 10.1038/s41598-017-06964-9.

Koo, H. Y. and Kume, T. (2013) 'FoxC1-Dependent Regulation of Vascular Endothelial Growth Factor Signaling in Corneal Avascularity', Trends in Cardiovascular Medicine. Elsevier, 23(1), p. 4. doi: 10.1016/j.tcm.2012.08.002.

Kulkeaw, K. and Sugiyama, D. (2012) 'Zebrafi sh erythropoiesis and the utility of fi sh as models of anemia', pp. 1-11.

Kume, T. et al. (1998) 'The forkhead/winged helix gene Mf1 is disrupted in the pleiotropic mouse mutation congenital hydrocephalus', Cell, 93(6), pp. 985-996. doi: 10.1016/S00928674(00)81204-0.

Kume, T. et al. (2001) 'The murine winged helix transcription factors, Foxc1 and Foxc2, are both required for cardiovascular development and somitogenesis', Genes and Development, 15(18), pp. 2470-2482. doi: 10.1101/gad.907301.

Lamont, R. E. et al. (2010) 'Hedgehog signaling via angiopoietin1 is required for developmental vascular stability.', Mechanisms of development. Ireland, 127(3-4), pp. 159-168. doi: 10.1016/j.mod.2010.02.001.

Lauter, G., Soll, I. and Hauptmann, G. (2011) 'Two-color fluorescent in situ hybridization in the embryonic zebrafish brain using differential detection systems', BMC developmental biology, 11, 
p. 43. doi: 10.1186/1471-213X-11-43.

Lee, M. Y. et al. (2015) 'Smooth muscle cell genome browser: Enabling the identification of novel serum response factor target genes', PLoS ONE, 10(8), pp. 1-22. doi:

10.1371/journal.pone.0133751.

Liao, Y., Smyth, G. K. and Shi, W. (2014) 'FeatureCounts: An efficient general purpose program for assigning sequence reads to genomic features', Bioinformatics, 30(7), pp. 923-930. doi: 10.1093/bioinformatics/btt656.

Lindahl, P. et al. (1997) 'Pericyte Loss and Microaneurysm Formation in PDGF-B-Deficient Mice', Science, 277(5323), pp. 242-245. doi: 10.1126/science.277.5323.242.

Liu, J. et al. (2007) 'A betaPix Pak2a signaling pathway regulates cerebral vascular stability in zebrafish', Proc Natl Acad Sci U S A, 104(35), pp. 13990-13995. doi:

10.1073/pnas.0700825104.

Love, M. I., Huber, W. and Anders, S. (2014) 'Moderated estimation of fold change and dispersion for RNA-seq data with DESeq2', Genome Biology, 15(12). doi: 10.1186/s13059-0140550-8.

Majesky, M. W. (2007) 'Developmental basis of vascular smooth muscle diversity', Arteriosclerosis, Thrombosis, and Vascular Biology, 27(6), pp. 1248-1258. doi:

10.1161/ATVBAHA.107.141069.

Mears, A. J. et al. (1998) 'Mutations of the forkhead/winged-helix gene, FKHL7, in patients with Axenfeld-Rieger anomaly.', American journal of human genetics, 63(5), pp. 1316-28. doi: 10.1086/302109.

Miesfeld, J. B. and Link, B. A. (2014) 'Establishment of transgenic lines to monitor and manipulate Yap/Taz-Tead activity in zebrafish reveals both evolutionarily conserved and divergent functions of the Hippo pathway.', Mechanisms of development. Ireland: Elsevier Ireland Ltd, 133(February), pp. 177-188. doi: 10.1016/j.mod.2014.02.003.

Mishra, S. et al. (2016) 'Cerebrovascular defects in Foxc1 mutants correlate with aberrant WNT and VEGF???A pathways downstream of retinoic acid from the meninges', Developmental Biology. Elsevier, 420(1), pp. 148-165. doi: 10.1016/j.ydbio.2016.09.019.

Mongera, A. et al. (2013) 'Genetic lineage labeling in zebrafish uncovers novel neural crest contributions to the head, including gill pillar cells', Development, 140(4), pp. 916-925. doi: 
10.1242/dev.091066.

Moura, D. A. P., Lemos, R. R. and Oliveira, J. R. M. (2017) 'New Data from Pdfgbret/retMutant Mice Might Lead to a Paradoxical Association Between Brain Calcification, Pericytes Recruitment and BBB Integrity', Journal of Molecular Neuroscience. Journal of Molecular Neuroscience, 63(3-4), pp. 419-421. doi: 10.1007/s12031-017-0992-z.

Nishimura, D. Y. et al. (1998) 'The forkhead transcription factor gene FKHL7 is responsible for glaucoma phenotypes which map to 6p25', Nature Genetics, 19(2), pp. 140-147. doi: 10.1038/493.

Olson, L. E. and Soriano, P. (2011) 'PDGFR?? signaling regulates mural cell plasticity and inhibits fat development', Developmental Cell. Elsevier Inc., 20(6), pp. 815-826. doi: 10.1016/j.devcel.2011.04.019.

Omatsu, Y. et al. (2014) 'Foxc1 is a critical regulator of haematopoietic stem/progenitor cell niche formation', Nature. Nature Publishing Group, 508(7497), pp. 536-540. doi: 10.1038/nature13071.

Pouget, C., Pottin, K. and Jaffredo, T. (2008) 'Sclerotomal origin of vascular smooth muscle cells and pericytes in the embryo', Developmental Biology, 315(2), pp. 437-447. doi: 10.1016/j.ydbio.2007.12.045.

Prasitsak, T. et al. (2015) 'Foxc1 is required for early stage telencephalic vascular development', Developmental Dynamics, 244(5), pp. 703-711. doi: 10.1002/dvdy.24269.

Proulx, K., Lu, A. and Sumanas, S. (2010) 'Cranial vasculature in zebrafish forms by angioblast cluster-derived angiogenesis', Developmental Biology. Elsevier Inc., 348(1), pp. 34-46. doi: 10.1016/j.ydbio.2010.08.036.

Qiu, J. et al. (2016) 'Embryonic hematopoiesis in vertebrate somites gives rise to definitive hematopoietic stem cells', Journal of molecular cell biology, 8(4), pp. 288-301. doi:

10.1093/jmcb/mjw024.

Quillien, A. et al. (2017) 'Robust Identification of Developmentally Active Endothelial Enhancers in Zebrafish Using FANS-Assisted ATAC-Seq', Cell Reports. ElsevierCompany., 20(3), pp. 709720. doi: 10.1016/j.celrep.2017.06.070.

Rougeot, J. et al. (2014) 'Host-Bacteria Interactions’, 1197. doi: 10.1007/978-1-4939-1261-2. 
Santoro, M. M., Pesce, G. and Stainier, D. Y. (2009) 'Characterization of vascular mural cells during zebrafish development', Mechanisms of Development. Elsevier Ireland Ltd, 126(8-9), pp. 638-649. doi: 10.1016/j.mod.2009.06.1080.

Sasman, A. et al. (2012) 'Generation of conditional alleles for Foxc1 and Foxc2 in mice', Genesis, 50(10), pp. 766-774. doi: 10.1002/dvg.22036.

Schildmeyer, L. a et al. (2000) 'Impaired vascular contractility and blood pressure homeostasis in the smooth muscle alpha-actin null mouse.', The FASEB journal : official publication of the Federation of American Societies for Experimental Biology, 14(14), pp. 2213-2220. doi: 10.1096/fj.99-0927com.

Schilling, T. F. et al. (1996) 'Jaw and branchial arch mutants in zebrafish II: anterior arches and cartilage differentiation', Development, 123(1), pp. 345-356. Available at: http://dev.biologists.org/content/123/1/345.short.

Schindelin, J. et al. (2012) 'Fiji: An open-source platform for biological-image analysis', Nature Methods, 9(7), pp. 676-682. doi: 10.1038/nmeth.2019.

Seiler, C., Abrams, J. and Pack, M. (2010) 'Characterization of zebrafish intestinal smooth muscle development using a novel sm22a-b promoter.', Developmental dynamics : an official publication of the American Association of Anatomists, 239(11), pp. 2806-2812. doi:

10.1002/dvdy.22420.

Seo, S. et al. (2006) 'The forkhead transcription factors, Foxc1 and Foxc2, are required for arterial specification and lymphatic sprouting during vascular development', Developmental Biology, 294(2), pp. 458-470. doi: 10.1016/j.ydbio.2006.03.035.

Seo, S. et al. (2012) 'Forkhead box transcription factor FoxC1 preserves corneal transparency by regulating vascular growth', Proceedings of the National Academy of Sciences, 109(6), pp. 2015-2020. doi: 10.1073/pnas.1109540109.

Seo, S. et al. (2017) 'Foxc1 and foxc2 in the neural crest are required for ocular anterior segment development', Investigative Ophthalmology and Visual Science, 58(3), pp. 1368-1377. doi: $10.1167 /$ iovs.16-21217.

Seo, S. and Kume, T. (2006) 'Forkhead transcription factors, Foxc1 and Foxc2, are required for the morphogenesis of the cardiac outflow tract', Developmental Biology, 296(2), pp. 421-436. doi: 10.1016/j.ydbio.2006.06.012. 
Siegenthaler, J. A. et al. (2013) 'Foxc1 is required by pericytes during fetal brain angiogenesis', Biology Open. England, 2(7), pp. 647-659. doi: 10.1242/bio.20135009.

Skarie, J. M. and Link, B. A. (2009) 'FoxC1 is essential for vascular basement membrane integrity and hyaloid vessel morphogenesis.', Investigative ophthalmology \& visual science. United States, 50(11), pp. 5026-5034. doi: 10.1167/iovs.09-3447.

Solway, J. et al. (1995) 'Structure and expression of a smooth muscle cell-specific gene, SM22a', Journal of Biological Chemistry, pp. 13460-13469. doi: 10.1074/jbc.270.22.13460.

Souzeau, E. et al. (2017) 'Glaucoma spectrum and age-related prevalence of individuals with FOXC1 and PITX2 variants', European Journal of Human Genetics, 2559(10), pp. 839-847. doi: 10.1038/ejhg.2017.59.

Van Steensel, M. A. M. et al. (2009) 'Novel missense mutations in the FOXC2 gene alter transcriptional activity', Human Mutation, 30(12), pp. 1002-1009. doi: 10.1002/humu.21127.

Stratman, A. N. et al. (2009) 'Pericyte recruitment during vasculogenic tube assembly stimulates endothelial basement membrane matrix formation', Blood, 114(24), pp. 5091-5101. doi:

10.1182/blood-2009-05-222364.

Stratman, A. N. et al. (2017) 'Interactions between mural cells and endothelial cells stabilize the developing zebrafish dorsal aorta', Development, 144(1), pp. 115-127. doi:

10.1242/dev.143131.

Tamimi, Y. et al. (2006) 'FGF19 is a target for FOXC1 regulation in ciliary body-derived cells', Human Molecular Genetics, 15(21), pp. 3229-3240. doi: 10.1093/hmg/ddl400.

Tao, Y. K. et al. (2017) 'Notch3 deficiency impairs coronary microvascular maturation and reduces cardiac recovery after myocardial ischemia', International Journal of Cardiology. Elsevier Ireland Ltd, 236, pp. 413-422. doi: 10.1016/j.jjcard.2017.01.096.

Topczewska, J. M. et al. (2001) 'Sequence and expression of zebrafish foxc1a and foxc1b, encoding conserved forkhead/winged helix transcription factors', Mechanisms of Development. Ireland, 100(2), pp. 343-347. doi: 10.1016/S0925-4773(00)00534-7.

Topczewska, J. M. et al. (2001) 'The winged helix transcription factor Foxc1a is essential for somitogenesis in zebrafish', Genes and Development, 15(18), pp. 2483-2493. doi:

10.1101/gad.907401. 
Trost, A. et al. (2016) 'Brain and Retinal Pericytes: Origin, Function and Role', Frontiers in Cellular Neuroscience, 10(February), pp. 1-13. doi: 10.3389/fncel.2016.00020.

Underly, R. G. et al. (2017) 'Pericytes as Inducers of Rapid, Matrix Metalloproteinase-9Dependent Capillary Damage during Ischemia', The Journal of Neuroscience, 37(1), pp. 129_ 140. doi: 10.1523/JNEUROSCI.2891-16.2017.

De Val, S. et al. (2008) 'Combinatorial Regulation of Endothelial Gene Expression by Ets and Forkhead Transcription Factors', Cell. Elsevier Ltd, 135(6), pp. 1053-1064. doi: 10.1016/j.cell.2008.10.049.

Vanlandewijck, M. et al. (2018) 'A molecular atlas of cell types and zonation in the brain vasculature', Nature. Nature Publishing Group, 554(7693), pp. 475-480. doi:

10.1038/nature25739.

Veldman, M. B. and Lin, S. (2012) 'Etsrp/Etv2 is directly regulated by Foxc1a/b in the zebrafish angioblast', Circulation Research, 110(2), pp. 220-229. doi:

\subsection{1/CIRCRESAHA.111.251298.}

Wang, Y. et al. (2014) 'Notch3 establishes brain vascular integrity by regulating pericyte number', Development, 141(2), pp. 307-317. doi: 10.1242/dev.096107.

Westerfield, M. (1995) 'The Zebrafish Book: A Guide for the Laboratory Use of Zebrafish (Danio Rerio).', in. Eugene, OR: University of Oregon Press.

Whitesell, T. R. et al. (2014) 'An a-smooth muscle actin (acta2/asma) zebrafish transgenic line marking vascular mural cells and visceral smooth muscle cells', PLoS ONE, 9(3), pp. 1-10. doi: 10.1371/journal.pone.0090590.

Winkler, E. A. et al. (2018) 'Reductions in brain pericytes are associated with arteriovenous malformation vascular instability', Journal of Neurosurgery, pp. 1-11. doi:

10.3171/2017.6.JNS17860.

Winkler, E. A., Bell, R. D. and Zlokovic, B. V (2010) 'Pericyte-specific expression of PDGF beta receptor in mouse models with normal and deficient PDGF beta receptor signaling', Molecular Neurodegeneration, 5(1), p. 32. doi: 10.1186/1750-1326-5-32.

Xu, P. et al. (2018) 'Fox proteins are modular competency factors for facial cartilage and tooth specification', (May). doi: 10.1242/dev.165498. 
Yamagishi, H. et al. (2003) 'Tbx1 is regulated by tissue-specific forkhead proteins through a common Sonic hedgehog-responsive enhancer', Genes and Development, 17(2), pp. 269-281. doi: 10.1101/gad.1048903.

Zarbalis, K. et al. (2007) 'Cortical dysplasia and skull defects in mice with a Foxc1 allele reveal the role of meningeal differentiation in regulating cortical development.', Proceedings of the National Academy of Sciences of the United States of America, 104(35), pp. 14002-7. doi: 10.1073/pnas.0702618104. 


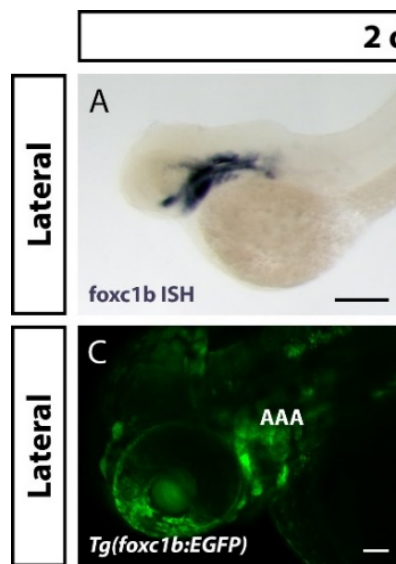

\section{$2 \mathrm{dpf}$}
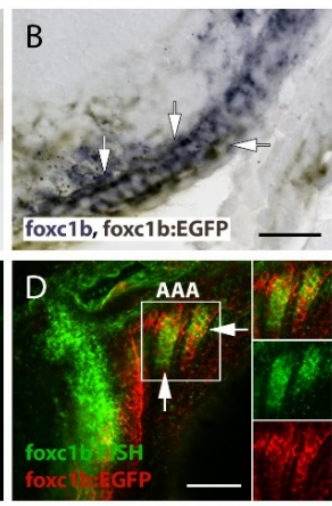

Tg(foxc1b:EGFP)
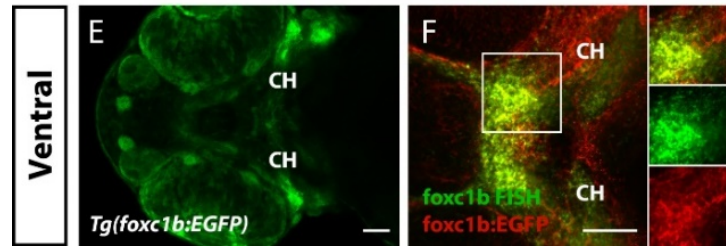

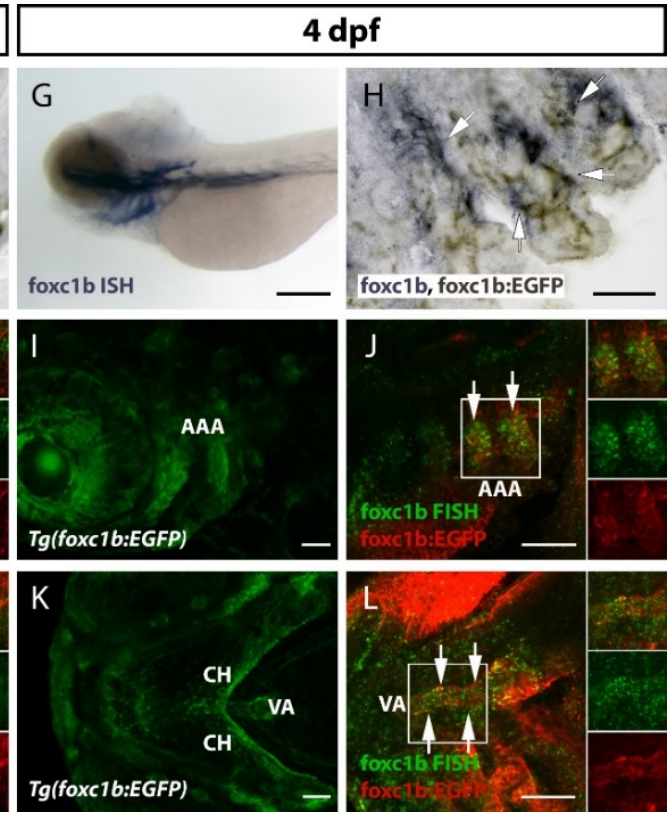

Figure 1: A foxc1b:EGFP transgenic line mimics endogenous foxc1b mRNA expression in the zebrafish ventral head. A-F) $2 \mathrm{dpf}$ and G-L) $4 \mathrm{dpf}$ images of foxc1b expression in lateral (A$\mathrm{D}, \mathrm{G}-\mathrm{J}$ ) and ventral views (E, F, K, L). A) foxc1b mRNA (purple) is expressed in the aortic arches, jaw, ceratohyal, and periocular mesenchyme of the ventral head of the zebrafish embryo. $B$ ) foxc $1 b$ mRNA and foxc1b:EGFP transgene expression (brown) overlap (arrows) in the ceratohyal. C) foxc1b:EGFP transgene (green) is strongly expressed in the aortic arches and mesenchyme of the ventral head. D) foxc1b mRNA expression (green) overlaps with foxc1b:EGFP antibody stain (red) in the aortic arch arteries (arrows). E) Strong expression of foxc1b:EGFP in the ventral head including the ceratohyal. F) foxc1b mRNA expression overlaps with foxc1b:EGFP antibody stain in the ceratohyal. G) At $4 \mathrm{dpf}$, foxc1b mRNA is present in the aortic arch arteries, the epibranchial region of the ventral brain, ceratohyal, swim bladder, and intestine. H) foxc1b mRNA and foxc1b:EGFP overlap in cells surrounding the aortic arch arteries. I) foxc1b:EGFP is expressed in aortic arch arteries. J) foxc1b mRNA expression overlaps with foxc1b:EGFP antibody stain in the aortic arch arteries (arrows). K) foxc $1 b$ :EGFP is expressed around the ventral aorta and ceratohyal. L) foxc1b mRNA expression overlaps with foxc1b:EGFP antibody stain in

614 the ventral aorta (arrows) and ceratohyal. Scale bars in A and G represent $200 \mu \mathrm{m}$. Scale bars in $615 \mathrm{~B}$ and $\mathrm{H}$ represent $20 \mu \mathrm{m}$. Scale bars in C-F and I-L represent $50 \mu \mathrm{m}$. Insets in D, F, J, and L 616 show merged, foxc1b:EGFP F-ISH, and foxc1b:EGFP antibody stain. AAA = aortic arch arteries, $\mathrm{CH}=$ ceratohyal, $\mathrm{VA}=$ ventral aorta. 

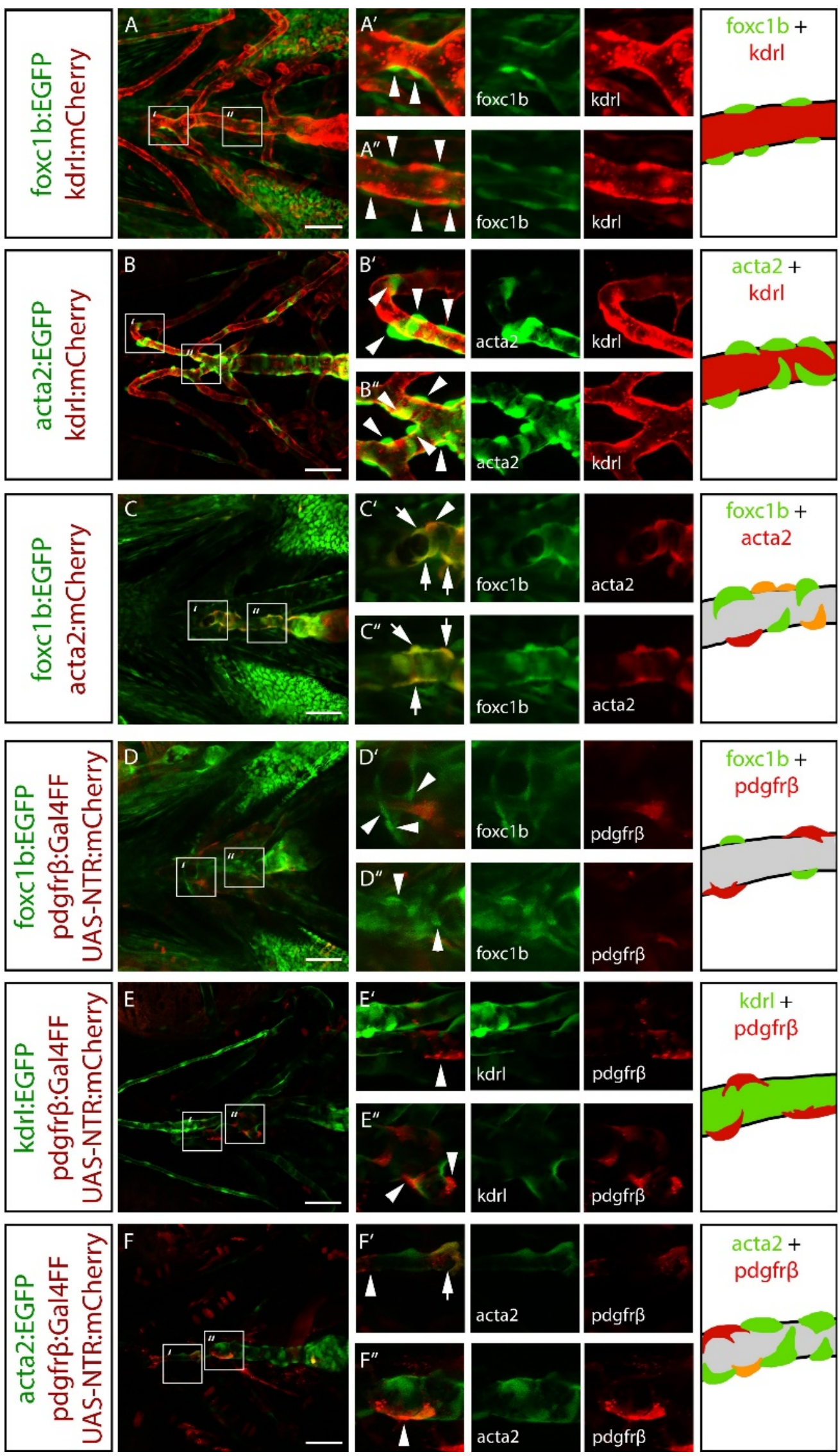
619 Figure 2: foxc1b:EGFP positive cells co-express a smooth muscle marker, but not

620 endothelial or pericyte markers. Vessels of the ventral head in $4 \mathrm{dpf}$ zebrafish. Arrowheads

621 represent marker-expressing mural cells. Arrows show co-expression of two mural cell markers.

622 A) foxc1b:EGFP expressing cells are associated with and surround the endothelium

623 (kdrl:mCherry) along the ventral aorta. B) acta2:EGFP positive smooth muscle cells surround the

624 kdrl:mCherry positive endothelium along the ventral aorta and aortic arch arteries. C)

625 foxc1b:EGFP and acta2:mCherry are co-expressed along the ventral aorta. D) foxc1b:EGFP is

626 not co-expressed in cells expressing a pericyte marker (pdgfr $\beta$ :mCherry) in the ventral head. E)

627 pdgfr $\beta$ :mCherry is associated with the endothelium ( $k d r l: E G F P$ ) in the ventral head. F)

628 pdgfrß:mCherry and acta2:EGFP are partially co-expressed in ventral head mural cells.

629 Schematics depict mural cell and endothelial marker expression that match the transgenes. Grey

630 indicates presumptive endothelial patterns. Scale bars represent $50 \mu \mathrm{m}$. 
631

632

633

634

635

636

637

638

639

640

641

642
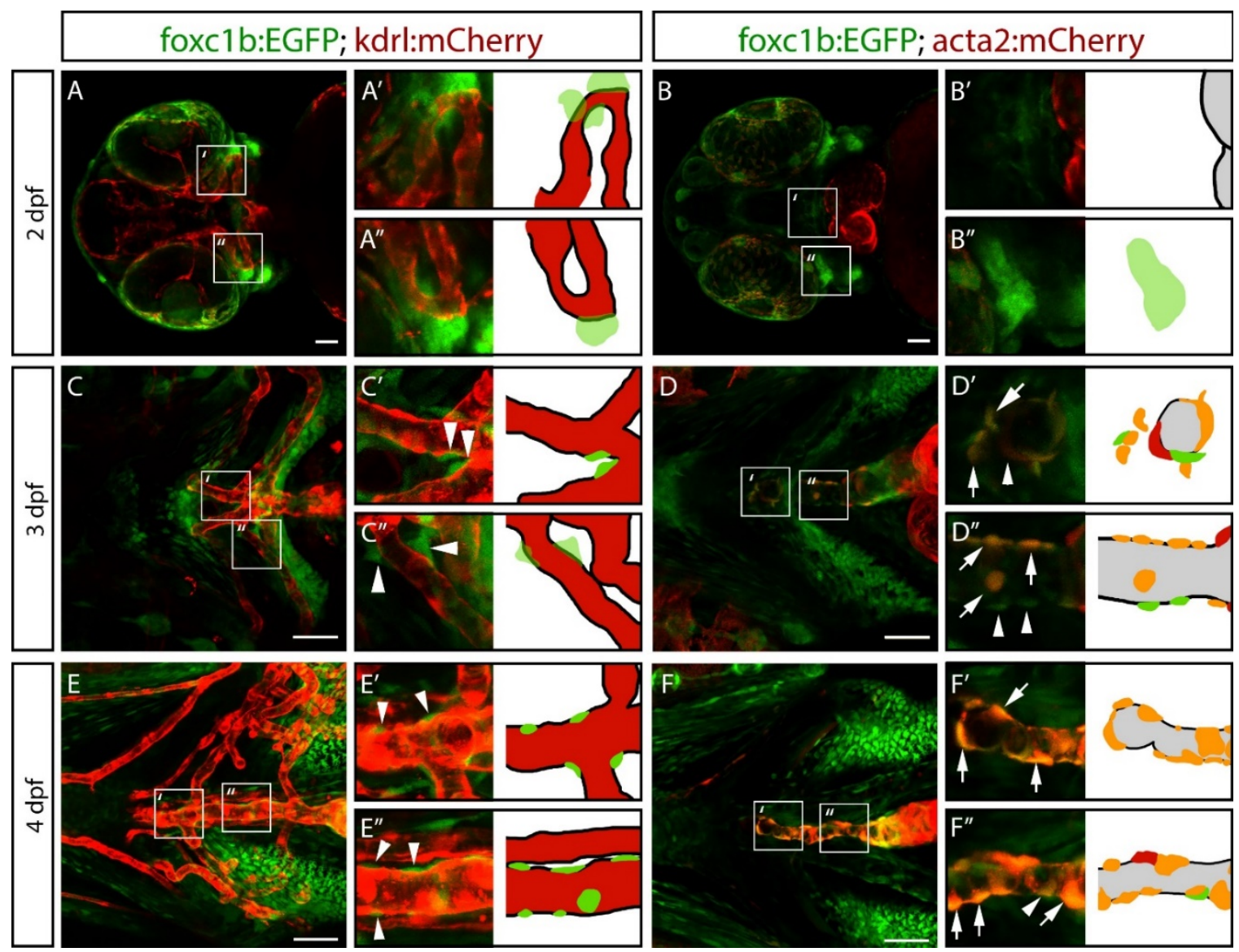
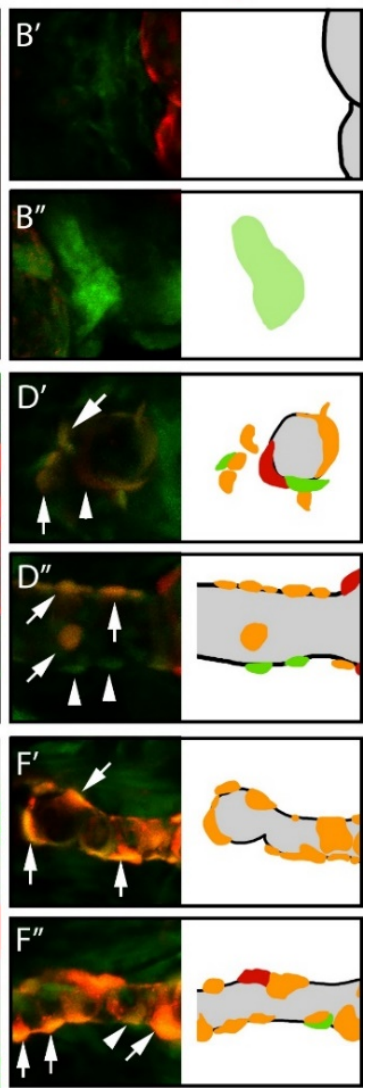

Figure 3: foxc1b:EGFP is expressed in mesenchymal cells as they associate with and attach to the endothelium. A, B) Images of the ventral head in 2 dpf zebrafish embryos show foxc1b:EGFP positive mesenchymal cells near the endothelium ( $k d r l: m$ Cherry; $\mathrm{n}=19$ embryos), but not co-expressing a smooth muscle marker (acta2:mCherry; $\mathrm{n}=6$ embryos), which is only expressed in the heart at this stage. C, D) At $3 \mathrm{dpf}$, foxc1b:EGFP positive cells associate with the endothelium ( $n=14$ embryos), and some cells co-express acta2:mCherry ( $n=5$ embryos). Arrowheads indicate a mural cell marker associated with the endothelium. Arrows indicate coexpression between two mural cell markers. E, F) At $4 \mathrm{dpf}$, foxc1b:EGFP positive cells associate with the endothelium ( $n=11$ embryos) and a greater proportion of foxc $1 \mathrm{~b}$ :EGFP positive cells coexpress acta2: $m$ Cherry ( $n=8$ embryos). Grey colour in schematics indicates endothelial patterns. Scale bars represent $50 \mu \mathrm{m}$. 

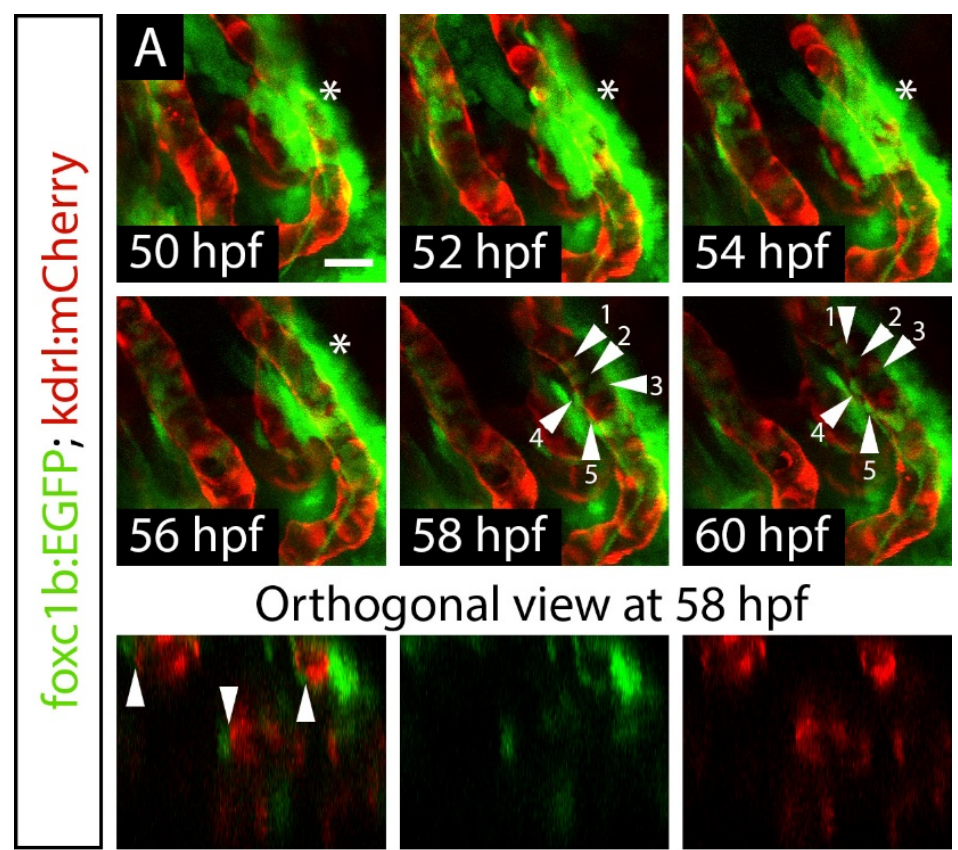

Orthogonal view at $58 \mathrm{hpf}$
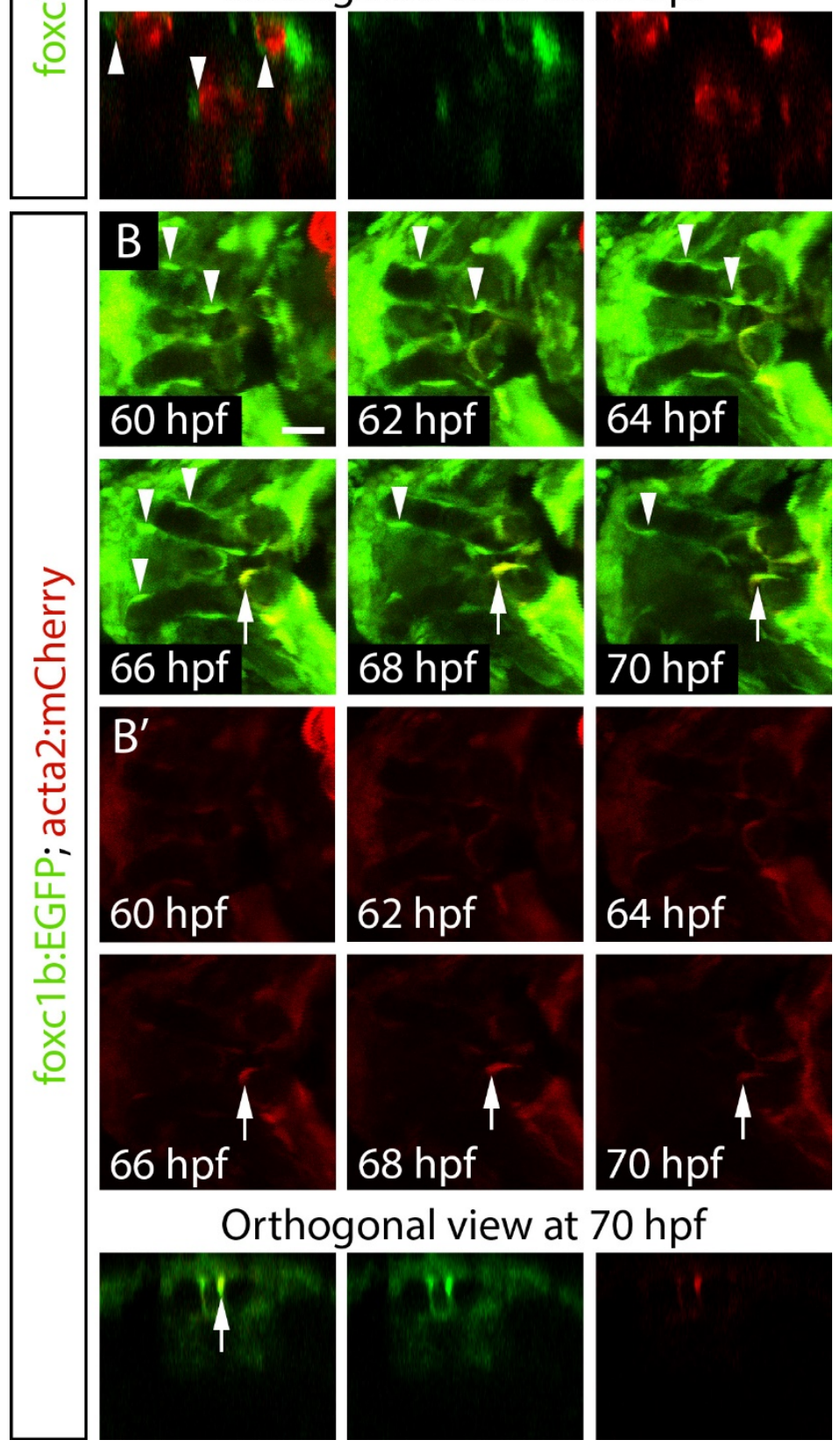

Orthogonal view at $70 \mathrm{hpf}$
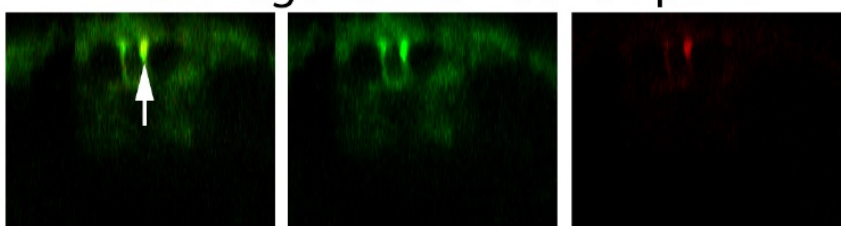

Figure 4: Time-lapse of foxc1b:EGFP positive mesenchymal cells associating with the endothelium and co-expressing a smooth muscle marker. A) Ventral views of aortic arch arteries of a foxc1b:EGFP; kdrl:mCherry embryo using confocal time-lapse imaging starting at $50 \mathrm{hpf}$. Mesenchymal cells are indicated by the asterisk, while vascular mural cells are indicated by arrowheads, starting by $56-58 \mathrm{hpf}$. Orthogonal views at $58 \mathrm{hpf}$ depict the foxc1b:EGFP cells wrapping around, but not co-expressing an endothelial marker. B) Time-lapse of the early ventral aorta of a foxc1b:EGFP; acta2:mCherry embryo from 60-70 hpf. foxc1b:EGFP positive smooth muscle cells are associated with the endothelium (arrowheads) but do not co-express acta2:mCherry until approximately 66 hpf (arrow). B') Single channel images of acta2:mCherry. Orthogonal views at 70 hpf show acta2:mCherry co-expressed within a foxc1b:EGFP positive cell. Scale bars represent $20 \mu \mathrm{m}$. 

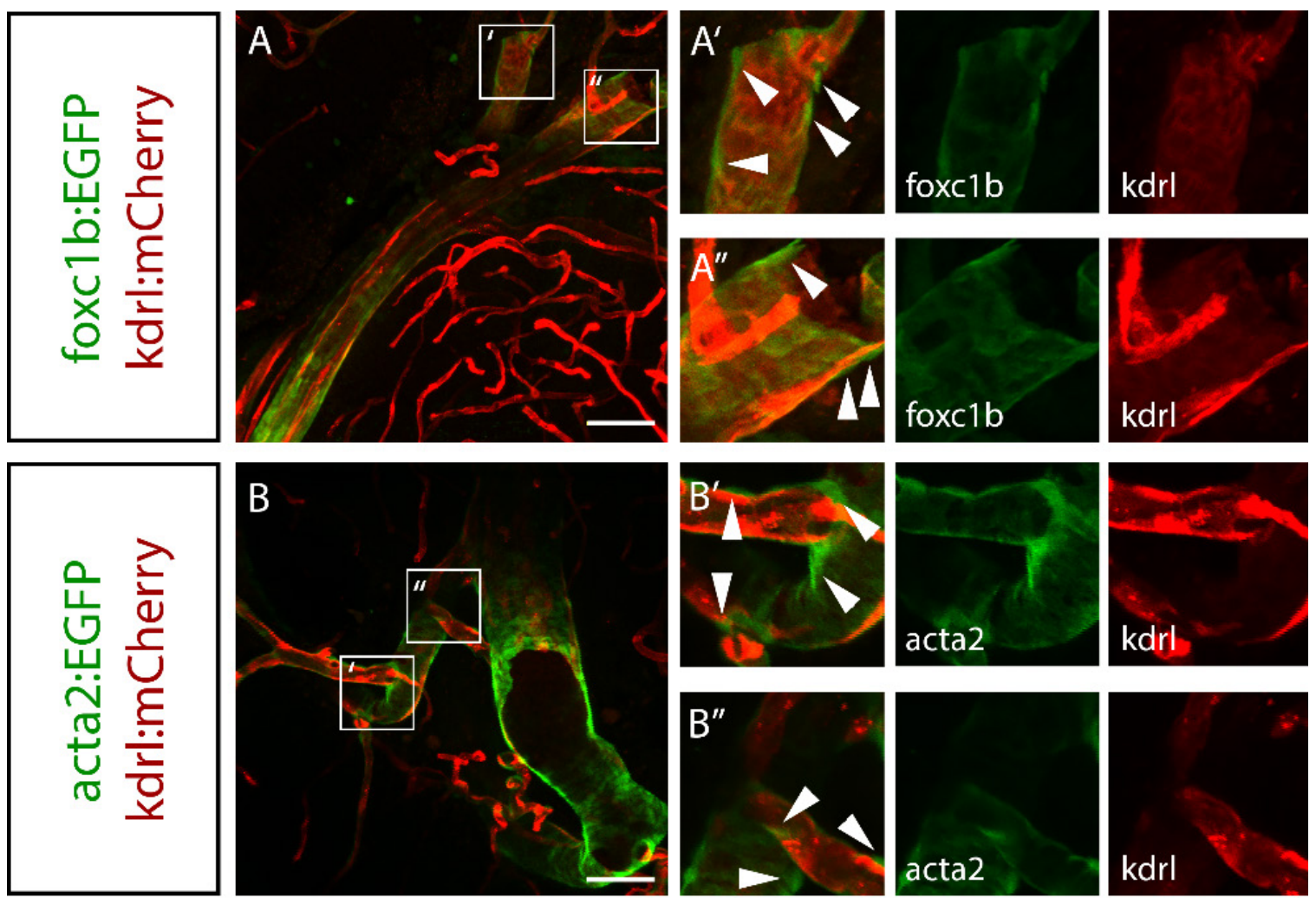

671
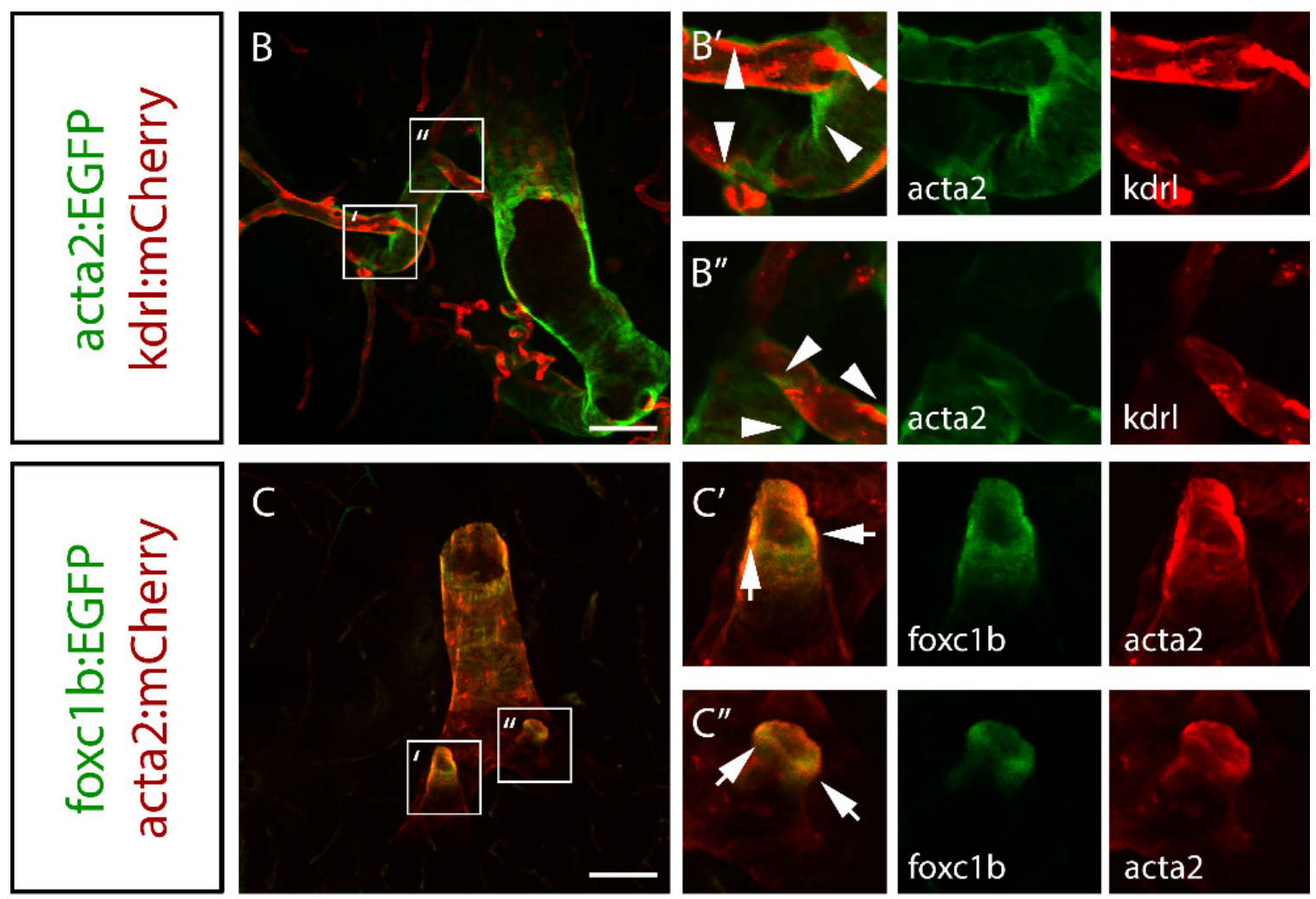

672 Figure 5: foxc1b:EGFP expression partially overlaps with acta2 expression in smooth

673 muscle cells in the adult zebrafish brain. A) foxc1b:EGFP expressing cells are closely

674 associated with endothelial cells ( $k d r l: m C h e r r y)$ in the adult brain. Arrowheads point to mural cells

675 associated with the endothelium. Arrows point to co-expression of two mural cell markers. B)

676 acta2:EGFP expressing smooth muscle cells are closely associated with kdrl:mCherry expressing

677 endothelial cells along large vessels of the brain. C) foxc1b:EGFP and acta2:mCherry are partially

678 co-expressed in smooth muscle cells on brain vessels. Scale bars represent $50 \mu \mathrm{m}$. 

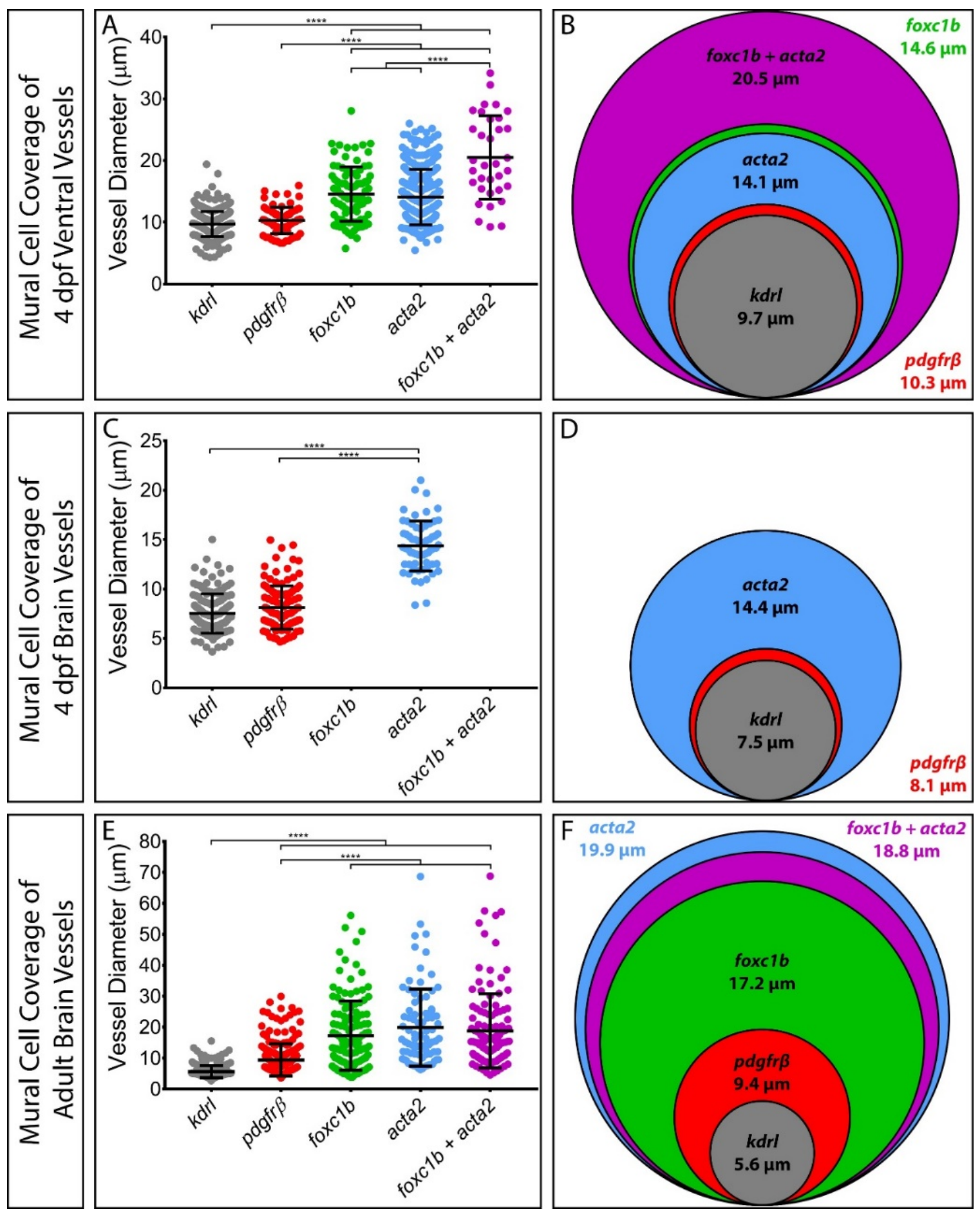

Figure 6: Mural cell marker expression is dependent upon vessel diameter, location, and developmental stage. A) Vessel diameters were measured from the endothelium adjacent to

682 mural cell markers at in the ventral head. Naked vessels are vessels labelled by the endothelial

$683 k d r l$ transgene only, while vessels covered in mural cells are categorized by the combination of 
684 mural cell markers expressed. Measurements of $4 \mathrm{dpf}$ ventral head vessels: $k d r l(9.7 \pm 2.0 \mu \mathrm{m}$, $685 \mathrm{n}=357), \operatorname{pdgfr} \beta(10.3 \pm 2.1 \mu \mathrm{m}, \mathrm{n}=75)$, foxc1b $(14.6 \pm 4.4 \mu \mathrm{m}, \mathrm{n}=89)$, acta2 $(14.1 \pm 4.5 \mu \mathrm{m}, \mathrm{n}=308)$, 686 and foxc1b + acta2 $(20.5 \pm 6.8 \mu \mathrm{m}, \mathrm{n}=34)$. B) Schematic of mean vessel diameter per condition 687 with vessel sizes scaled relative to each other. C) Measurements of $4 \mathrm{dpf}$ embryonic brain 688 vessels: $k d r l(7.5 \pm 2.0 \mu \mathrm{m}, \mathrm{n}=147)$, $\operatorname{pdgfr} \beta(8.1 \pm 2.2 \mu \mathrm{m}, \mathrm{n}=119)$, and acta2 $(14.4 \pm 2.5 \mu \mathrm{m}$, $689 \mathrm{n}=63)$. D) Schematic of mean vessel diameter per condition with vessel sizes scaled relative to 690 each other. E) Measurements of adult brain vessels: $k d r l(5.6 \pm 2.0 \mu \mathrm{m}, \mathrm{n}=244), \operatorname{pdgfr} \beta(9.4 \pm 5.2$ $691 \mu \mathrm{m}, \mathrm{n}=238)$, foxc1b (17.2 $\pm 11.2 \mu \mathrm{m}, \mathrm{n}=133)$, acta2 $(19.9 \pm 12.5 \mu \mathrm{m}, \mathrm{n}=83)$, and foxc1b + acta2 $692(18.8 \pm 12.0 \mu \mathrm{m}, \mathrm{n}=126)$. F) Schematic of mean vessel diameter per condition with vessel sizes 693 scaled relative to each other. ${ }^{* * *}=p<0.0001$, ANOVA with Tukey's multiple comparisons test. 

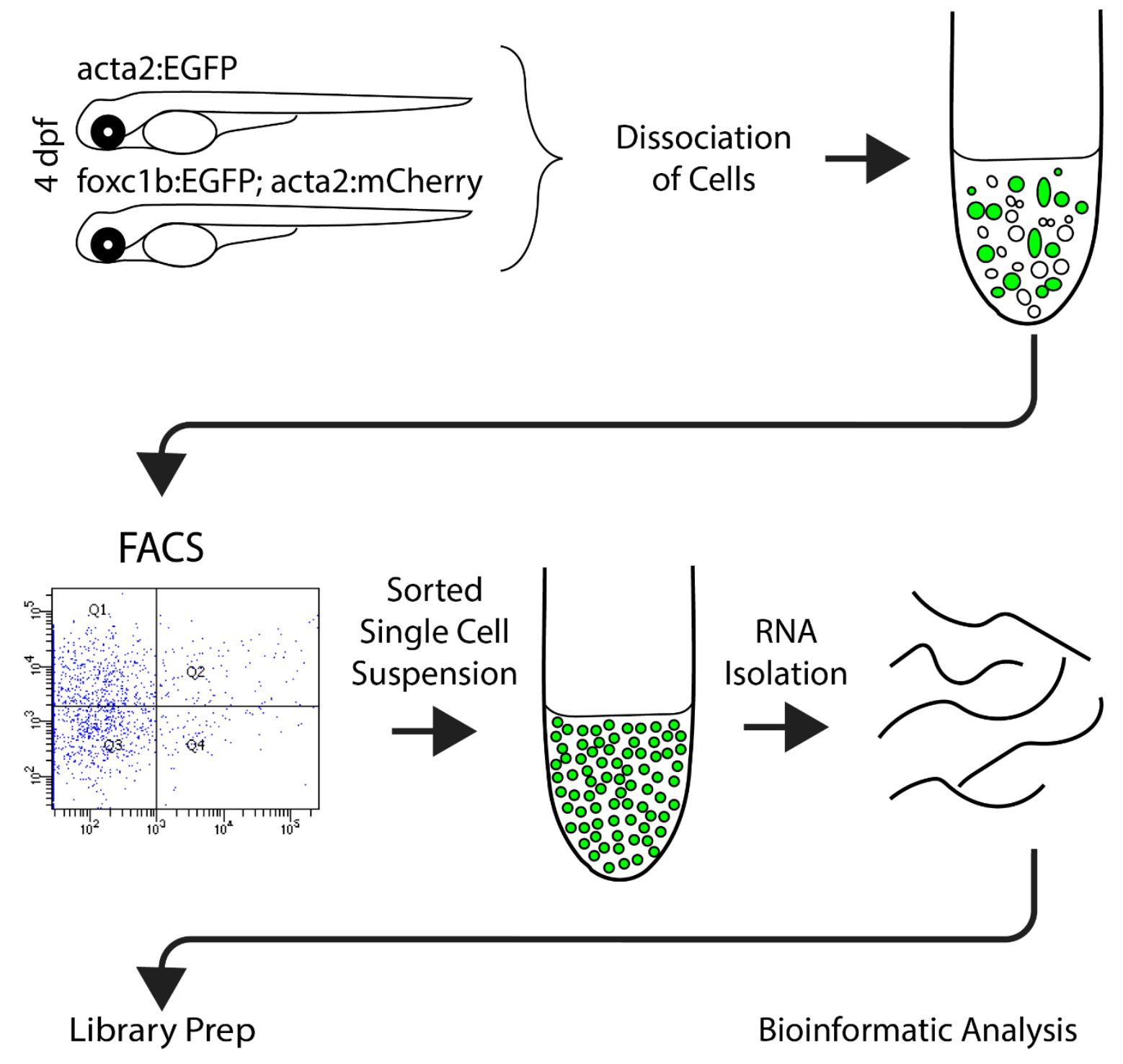

\section{andolo}

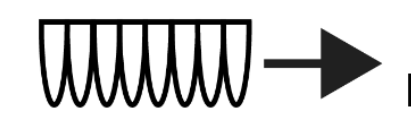

RNA Sequencing Illumina Next Seq

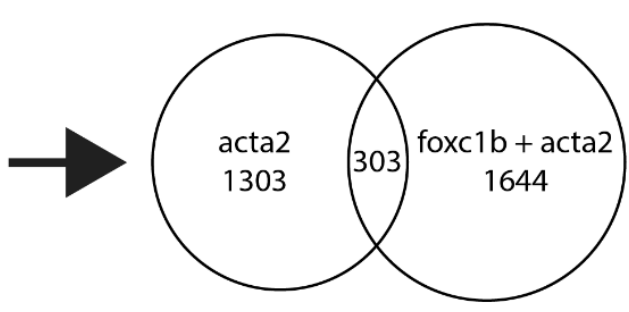

Figure 7: Workflow for isolating smooth muscle transcriptomes from zebrafish embryos. 4 and $5 \mathrm{dpf}$ zebrafish embryos were pooled and dissociated before fluorescence-activated cell sorting. RNA was isolated and sequenced using an Illumina Next Seq platform. 

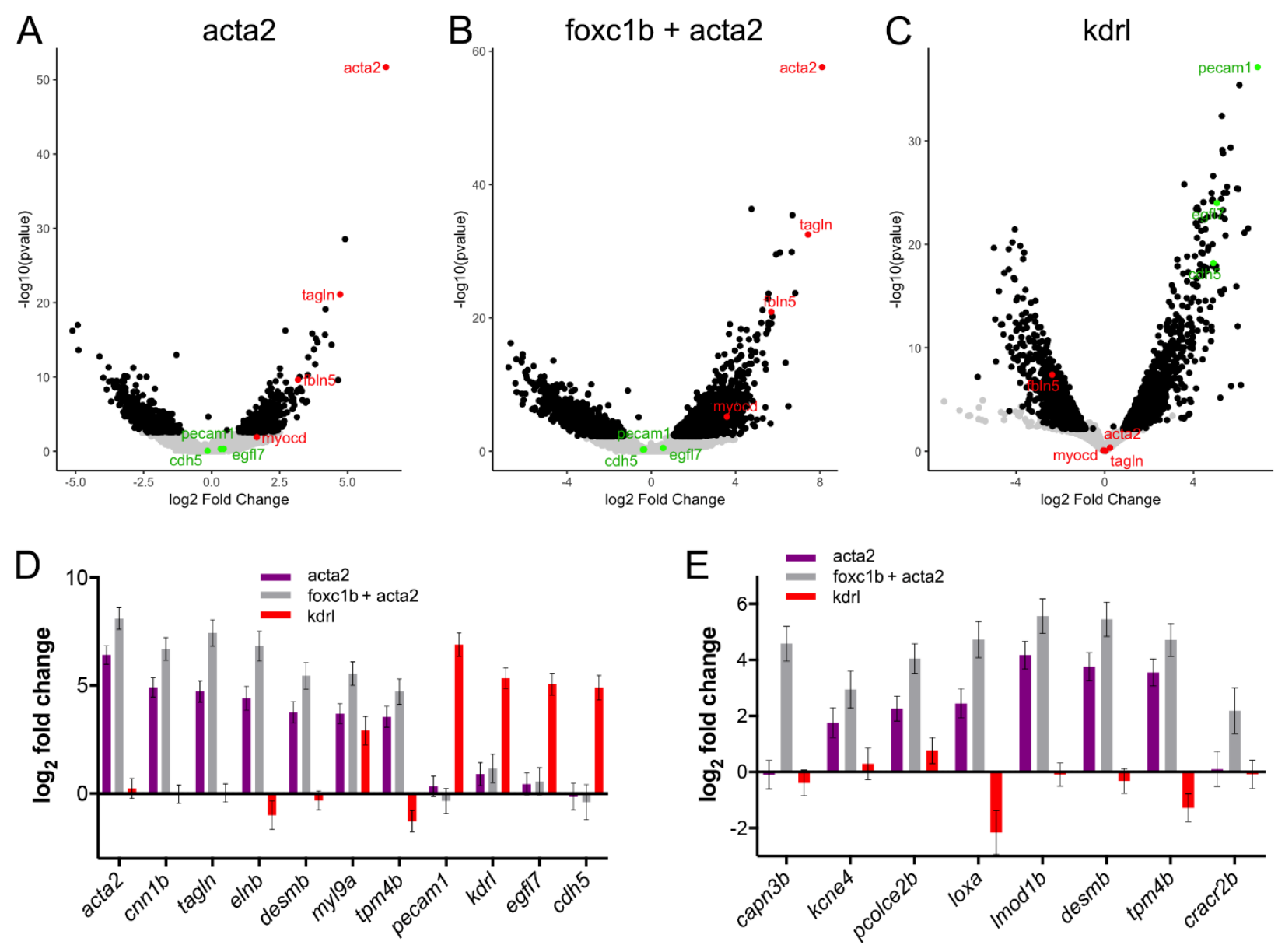

Figure 8: Differential gene expression in embryonic smooth muscle cells. A-C) Volcano plots for acta2 smooth muscle (A), foxc1b + acta2 smooth muscle (B), and endothelial (C) datasets. Representative smooth muscle genes are shown in red, whereas endothelial genes are shown in green. Black dots represent genes with significant changes ( $p$ adj < 0.05). D) $\log _{2}$ fold change in each dataset for genes indicated in the volcano plots. E) Log $_{2}$ fold change in each error. 


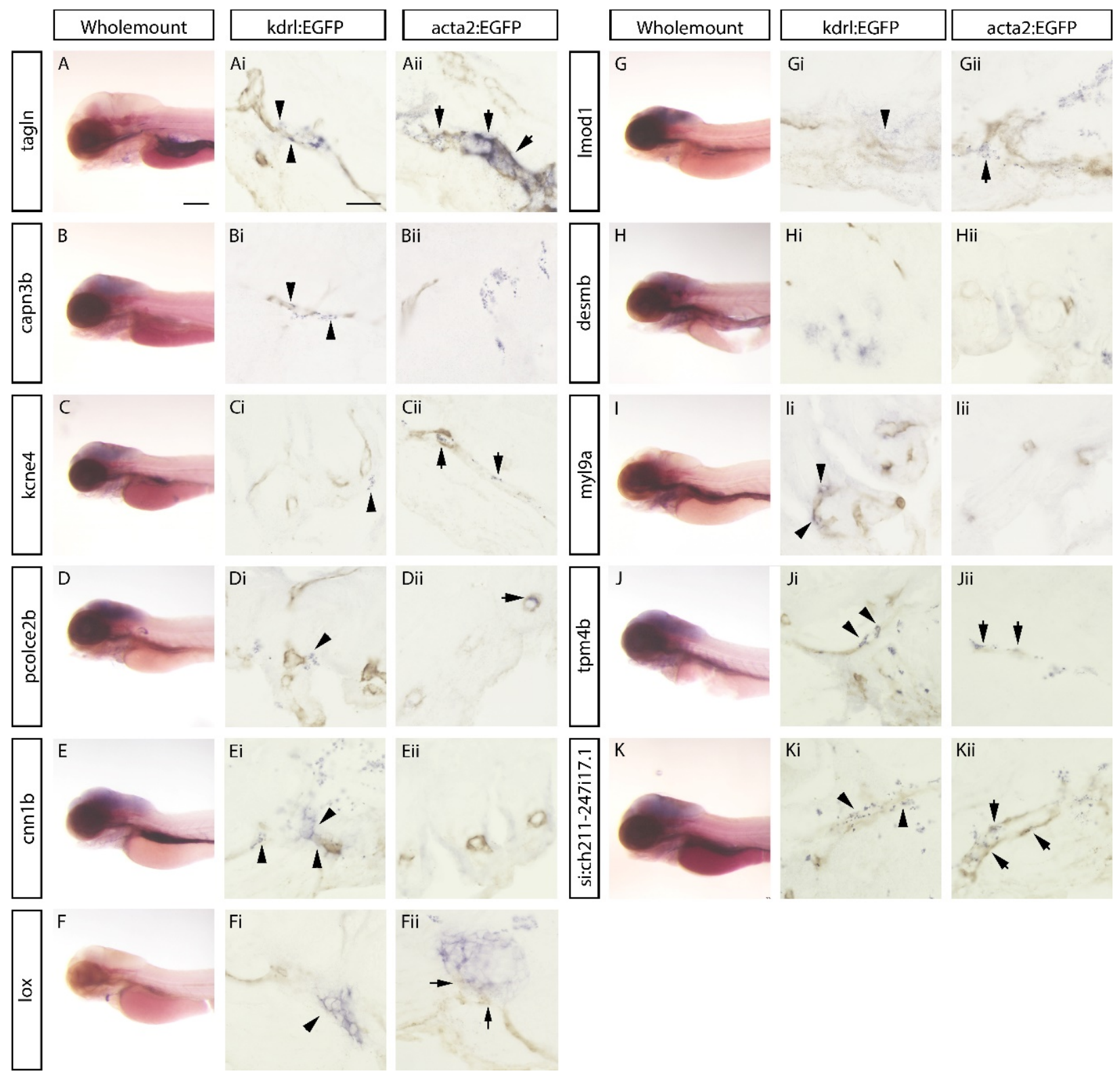

Figure 9: Validation of genes from the mural cell transcriptomes. Gene expression in lateral view wholemount and sections, compared to endothelial (antibody staining for kdrl:EGFP, brown, column i), or smooth muscle (acta2:EGFP, brown, column ii) transgenes. Arrowheads depict gene expression associated with the endothelium, arrows depict gene expression co-expressed with 711 smooth muscle. A) tagln, a known smooth muscle marker, is found in the aortic arch arteries, 712 notochord tip, swim bladder, and gut in wholemount. In sections tagln is expressed in cells 713 adjacent to the endothelium and is co-expressed with acta2:EGFP on the ventral aorta and aortic 714 arch arteries. B) capn $3 b$ is expressed in cells adjacent to the endothelium in the brain. C) kcne4 715 is expressed adjacent to the endothelium and is co-expressed in smooth muscle on the ventral 
716 aorta and aortic arch arteries. D) pcolce $2 b$ is expressed adjacent to the endothelium and is co-

717 expressed with acta2:EGFP. E) $c n n 1 b$ is expressed adjacent to the endothelium of the aortic arch

718 arteries. F) lox is expressed solely in the smooth muscle of the bulbus arteriosus. G) Imod1 is

719 expressed adjacent to the endothelium and overlaps with acta2:EGFP. H) desmb is expressed in

720 a punctate pattern in the brain. I) myl9a is expressed adjacent to the endothelium, and partially

721 overlaps with acta2:EGFP. J) tpm4b is expressed near the endothelium in the ventral head and

722 is co-expressed with acta2:EGFP in the brain. K) si:ch211-247i17.1 is expressed near the

723 endothelium and is co-expressed with acta2:EGFP. Scale bar for A-K (wholemount) represent

$724200 \mu \mathrm{m}$. Scale bars for sections (i and ii) represent $20 \mu \mathrm{m}$.

725 

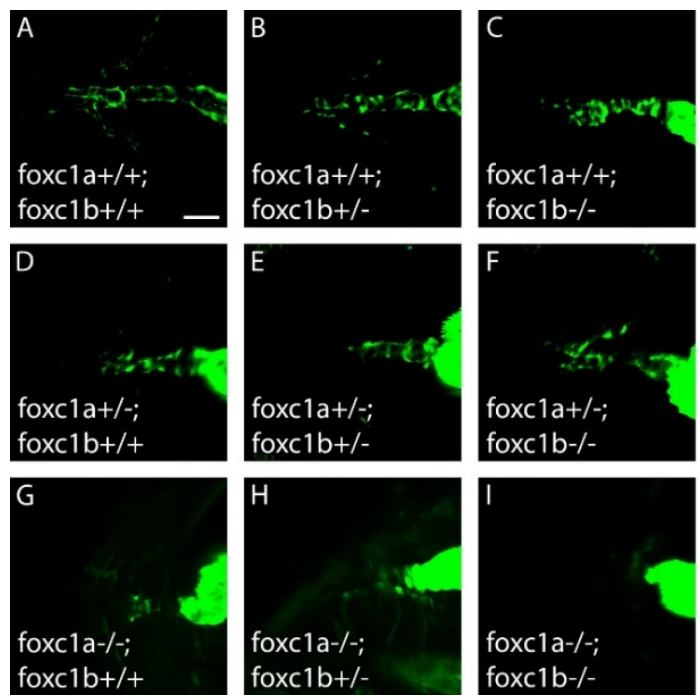

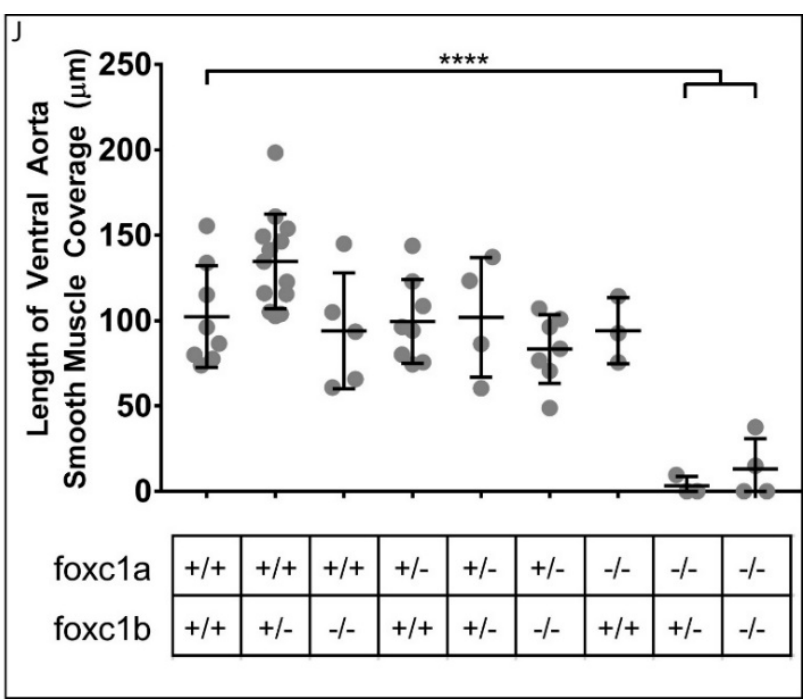

727 Figure 10: foxc1a/foxc1b mutants show increased loss of smooth muscle coverage with

728 the progressive loss of foxc1a/foxc1b alleles. Smooth muscle coverage (acta2:EGFP) at $4 \mathrm{dpf}$

729 on the ventral aorta of foxc1a and foxc1b compound mutants. Genotypes are listed in each

730 corresponding panel. A-C) foxc $1 a^{+/+}$fish show no statistical change in the length of ventral aorta

731 smooth muscle coverage, with progressive loss of foxc $1 b^{(+/+>+/->-/)}$. D-F) foxc1 $a^{+/-}$mutants show

732 no statistical change in the length of ventral aorta smooth muscle coverage with progressive loss

733 of $f \circ x c 1 b^{(+/+>+/>-/)}$. G-I) foxc1 $a^{-/-}$mutants show a statistical significant decrease in acta2:EGFP

734 positive cells on the ventral aorta in foxc $1 b^{+/-}$and foxc $1 b^{-/-}$mutants. J) Quantification of the length

735 of ventral aorta smooth muscle coverage, ${ }^{* * *}=p<0.0001$, ANOVA with Dunnett's multiple comparisons test. Scale bar represents $50 \mu \mathrm{m}$. 